\title{
Complexity in Surfaces of Densest Packings for Families of Polyhedra
}

\author{
Elizabeth R. Chen, ${ }^{1,2, *}$ Daphne Klotsa, ${ }^{2, \dagger}$ Michael Engel, ${ }^{2}$ Pablo F. Damasceno, ${ }^{3}$ and Sharon C. Glotzer ${ }^{2,3,4,+}$ \\ ${ }^{1}$ School of Engineering and Applied Sciences, Harvard University, Cambridge, Massachusetts 02138, USA \\ ${ }^{2}$ Department of Chemical Engineering, University of Michigan, Ann Arbor, Michigan 48109, USA \\ ${ }^{3}$ Applied Physics Program, University of Michigan, Ann Arbor, Michigan 48109, USA \\ ${ }^{4}$ Department of Materials Science and Engineering, University of Michigan, \\ Ann Arbor, Michigan 48109, USA
}

(Received 10 September 2013; revised manuscript received 20 December 2013; published 25 February 2014)

Packings of hard polyhedra have been studied for centuries due to their mathematical aesthetic and more recently for their applications in fields such as nanoscience, granular and colloidal matter, and biology. In all these fields, particle shape is important for structure and properties, especially upon crowding. Here, we explore packing as a function of shape. By combining simulations and analytic calculations, we study three two-parameter families of hard polyhedra and report an extensive and systematic analysis of the densest known packings of more than 55000 convex shapes. The three families have the symmetries of triangle groups (icosahedral, octahedral, tetrahedral) and interpolate between various symmetric solids (Platonic, Archimedean, Catalan). We find optimal (maximum) packing-density surfaces that reveal unexpected richness and complexity, containing as many as 132 different structures within a single family. Our results demonstrate the importance of thinking about shape not as a static property of an object, in the context of packings, but rather as but one point in a higher-dimensional shape space whose neighbors in that space may have identical or markedly different packings. Finally, we present and interpret our packing results in a consistent and generally applicable way by proposing a method to distinguish regions of packings and classify types of transitions between them.

DOI: 10.1103/PhysRevX.4.011024

\section{INTRODUCTION}

The optimization problem of how to pack objects in space as densely as possible has a long and colorful history [1-3]. Packing problems are both easy to grasp and notoriously hard to solve mathematically, qualities that have made them interesting recreational math puzzles [4]. Recent work on nanoparticle and colloidal self-assembly [5-7], micrometer molecule analogs [8], reconfigurability [9-17], and jammed granular matter [18,19], as well as biological cell aggregation [20,21] and crowding [22,23], has motivated further the study of packing. Packing in containers has a broad range of applications in operations research, such as optimal storage, packaging, and transportation $[24,25]$.

Despite significant progress in the study of packing, knowledge remains patchy and focuses on a few selected shapes with high symmetry. The densest packing of the sphere, known as the Kepler conjecture and formulated

\footnotetext{
*bethchen@umich.edu

†dklotsa@umich.edu

*sglotzer@umich.edu
}

Published by the American Physical Society under the terms of the Creative Commons Attribution 3.0 License. Further distribution of this work must maintain attribution to the author(s) and the published article's title, journal citation, and DOI.
Subject Areas: Materials Science, Soft Matter

over 400 years ago [26], was proven by Hales in 2005 $[1,27,28]$. Besides the sphere and objects that tile space (i.e., fill space completely without gaps or overlaps), no mathematical proofs have been found and results are obtained numerically, which means that other, denser packings may exist (e.g., aperiodic, large primitive unit cells). Throughout this paper, all of the densest packings reported or cited refer to the densest known packings.

Motivated by the great diversity of nanoparticle shapes that can now be synthesized $[7,29,30]$, many groups have studied dense packings of highly symmetric polyhedra (Platonic solids, Archimedean solids, and others) [31-38]. Yet, finding the densest (optimal) packing is challenging even for seemingly simple shapes such as the tetrahedron $[32-35,39,40]$. Recent experiments have gone even further by synthesizing nanoparticles-specifically, nanocubes (superballs) [13], whose shape can be tuned from a cube to an octahedron via a sphere-generating homologous families of shapes whose packings may vary with shape. To date, most theoretical and/or computational studies have reported the densest known packings for shape deformations of one-parameter families (i.e., one "axis" in "shape space"). Examples include ellipsoids [41], superballs [13,42], puffy tetrahedra [43], concave $n$-pods [36] and bowls [44], convex shapes characterized by aspect ratios [45], as well as truncated polyhedra, such as the tetrahedron-octahedron family [37], the octahedron-cube 
family [46], and tetrahedral dimers [38]. Many of these recent studies report a diversity of densest packings as a function of shape, resulting in a topographically complex line through what is actually a high-dimensional shape space. The behavior of higher-dimensional maximum-density surfaces in shape space obtained by varying two or more shape parameters simultaneously, as we do in this paper, affords a more in-depth look at the role of shape in packing. In particular, such a study allows for the identification and definition of topographical features (regions, types of boundaries). These definitions characterize the density surfaces and facilitate the comparison among different packing studies.

In this paper, we investigate the packing problem for three two-parameter families of symmetric convex polyhedra. Our families interpolate between edge-transitive polyhedra via continuous vertex and/or edge truncations. The interpolation goes through various solids (Platonic, Archimedean, Catalan), thereby including some of the above-referenced one-parameter studies as linear paths (subfamilies) on our two-parameter surfaces. The maximum packing density forms surfaces in shape space that reveal great diversity in richness and complexity. Our results demonstrate that some not previously studied paths through shape space give a plethora of consecutive distinct packing structures through a series of transitions (as the shape deforms), whereas other paths give the same or similar packings with no or few transitions. Given the richness of the surfaces of the densest packings, we aim to standardize the way packing results are presented and interpreted in the community by doing the following. Based on the theories of Minkowski [47,48] and the Kuperbergs [49], we define regions of topologically equivalent packings from their intersection equations (contacts with nearest neighbors) as opposed to their Bravais lattice type or symmetry group. We thus define and classify three types of boundaries between adjacent regions (valley, ridge, tangent). The classification is general for continuous families of shapes. We analyze previous works and argue that packing problems can be treated consistently using our framework.

The paper is organized as follows: Section II introduces some of the theoretical concepts and mathematical tools that have been formulated over the years for packing problems. We construct three two-parameter families of symmetric polyhedra in Sec. III. Section IV describes our analytical, numerical, and computational methods. We show results for the surfaces of maximum packing density in Sec. Vand close with a comparison with other studies in Sec. VI, and a summary of our main points and conclusion appear in Sec. VII.

\section{THEORETICAL BACKGROUND}

The packing problem is an optimization problem that searches for the densest possible packing arrangement of objects $\Xi$ in a container or in infinite Euclidean space. It is, in general, an intractable problem that does not allow a rigorous analytic treatment or numerical search. When packing in containers, the problem depends on a finite number of object positions and orientations and can often be solved via a brute-force search [50]. Packing in infinite space requires the optimization of an infinite number of variables. Here, we focus on the packing of identical convex objects (convex particles) in infinite Euclidean space. Because all known densest packings of convex objects are periodic, we restrict our search to those. The packing density of a periodic packing is $\phi=n U / V$, where $U$ is the volume of the object, $V$ is the volume of the unit cell of the lattice, and $n$ is the number of objects in the unit cell.

In this section, we give a theoretical background on mathematical concepts, tools, and theories that have been developed to solve packing problems and that we use in our approach in this paper. Specifically, we introduce (i) definitions for the sum and difference bodies (from set theory), where polyhedra are defined as sets of points comprising the shape, (ii) Minkowski's theory on packings [47,48] that have one particle in the primitive unit cell, and (iii) the Kuperbergs' extension of Minkowski's theory to include packings that have two antiparallel particles in the primitive unit cell [49].

\section{A. Sum and difference bodies}

Given two particles $\Xi$ and $Z$, the sum body and the difference body are the sets of sums and differences of all points in the particles:

$$
\begin{aligned}
& \Xi+Z=\{\xi+\zeta: \xi \in \Xi, \zeta \in Z\} \\
& \Xi-Z=\{\xi-\zeta: \xi \in \Xi, \zeta \in Z\} .
\end{aligned}
$$

See Fig. 1 for an example of the sum and difference bodies for a noncentrally symmetric polyhedron.

For any particle $\Xi$, we define positive orientation as $+\Xi=\Xi$ and negative orientation $-\Xi$ by inversion at the origin. Two particles $\Xi$ and $Z$ are parallel, if there exists a vector $\varsigma$ such that $Z=\varsigma+\Xi$. They are antiparallel, if there exists a vector $\varsigma$ such that $Z=\varsigma-\Xi$. Two particles $\Xi$ and $Z$ are in contact, if their intersection is equal to the intersection of their boundaries $\Xi \cap Z=\partial \Xi \cap \partial Z$. From basic set theory, we know the following.

(i) If a convex particle $\Xi$ (centered at 0 ) and a parallel neighbor $\varsigma+\Xi$ (centered at $\varsigma$ ) touch, then $\varsigma$ lives on the surface of the difference body $\Xi-\Xi$ (see the Appendix, Fig. 11, top left and right).

(ii) If a convex particle $\Xi$ (centered at 0 ) and an antiparallel neighbor $\varsigma-\Xi$ (centered at $\varsigma$ ) touch, then $\varsigma$ lives on the surface of the sum body $\Xi+\Xi$ (see the Appendix, Fig. 11, bottom left and right).

(iii) The sum body of particle $\Xi$ is always convex, even if $\Xi$ is not. 

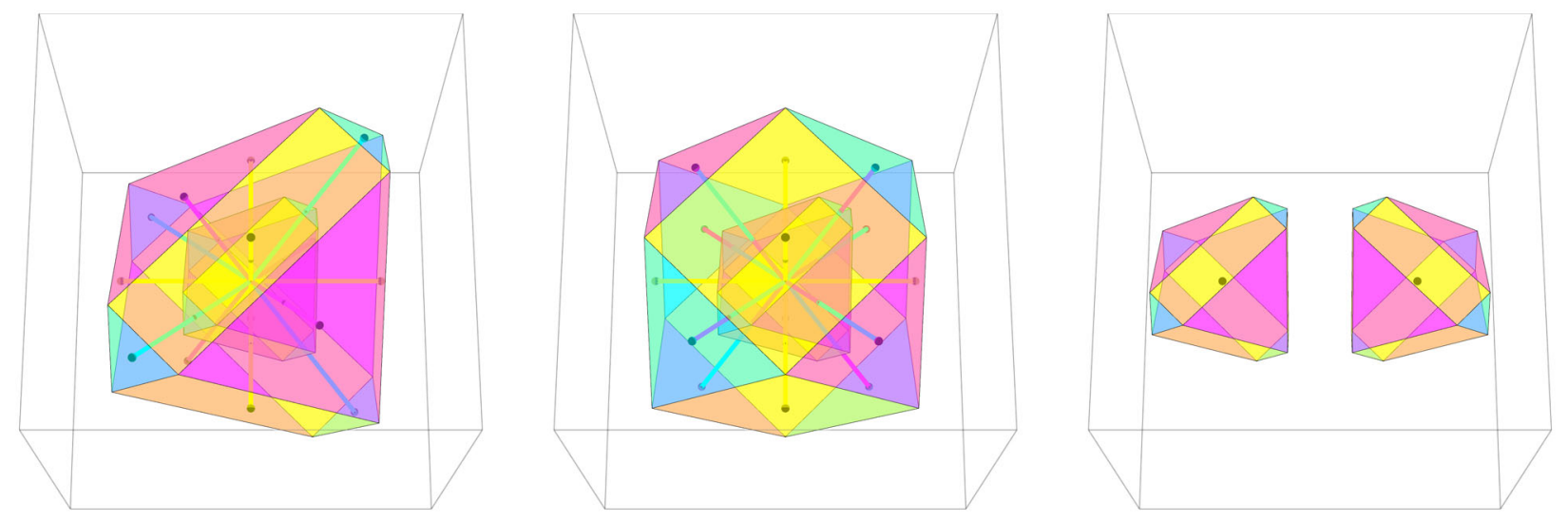

FIG. 1 The sum body (left) and difference body (middle) for a noncentrally symmetric polyhedron. A Kuperberg pair for the same polyhedron (right).

(iv) For a convex particle $\Xi$, the sum body has the same shape but twice the size: $\Xi+\Xi=2 \Xi$ (see Fig. 1, left).

(v) The difference body of particle $\Xi$ is always centrally symmetric, even if $\Xi$ is not (see Fig. 1, middle).

(vi) For a centrally symmetric particle $\Xi$, the difference body equals the sum body.

Examples of parallel and antiparallel neighbors of a noncentrally symmetric polyhedron, whose centers are on the difference and sum bodies, are shown in the Appendix, Fig. 11.

\section{B. Minkowski lattices}

A lattice packing is a packing with one particle in the primitive unit cell $(n=1)$. It has been observed that the densest known packing for many convex particles with central symmetry is a lattice packing [33,36-38], but this is not generally true [41]. The densest packings of noncentrally symmetric shapes frequently require two or more particles in the primitive unit cell.

The theory of lattice packings was originally developed by Minkowski $[47,48]$, who described packings considering the contacts of a particle with its neighbors. Chen [51] used this method to study the densest packings of various shapes. In a lattice packing, all particles are parallel and have identical neighborhoods related by translations, which are linear combinations with integer coefficients of the lattice vectors $\{\chi, \psi, \omega\}$. Minkowski proved the following.

(1) The densest lattice packing (Minkowski lattice) of a convex particle $\Xi$ is always identical to the densest lattice packing of its difference body $\Xi-\Xi$.

This theorem is important because it is easier to (i) mathematically manipulate centrally symmetric shapes and (ii) write down intersection equations in terms of the difference body [see Sec. IV B and the Supplemental Material [52] (p. 6-42)].

(2) For all centrally symmetric convex shapes (in three dimensions), a Minkowski lattice can always be chosen such that each particle $\Xi$ is in contact with either 12 or 14 neighbors and the lattice satisfies one of three possible types with either six or seven pairs of neighbor contacts:

$$
\begin{aligned}
& G^{6-}= \pm\{\chi, \psi, \omega, \psi-\omega, \omega-\chi, \chi-\psi\} \\
& G^{6+}= \pm\{\chi, \psi, \omega, \psi+\omega, \omega+\chi, \chi+\psi\}, \\
& G^{7+}= \pm\{\chi, \psi, \omega, \psi+\omega, \omega+\chi, \chi+\psi, \chi+\psi+\omega\} .
\end{aligned}
$$

Examples of the Minkowski lattice types $G^{6-}, G^{7+}$, and $G^{6+}$ for a noncentrally symmetric polyhedron are shown in Fig. 2.

We make two comments regarding the relation of the Minkowski lattices (and all packings reported in this paper) to Bravais lattices employed in crystallography. First, in connection to the unit cell, we choose vectors that form a basis for the lattice with a minimum positive determinant (lattice volume), and thus we always use the primitive unit cell (smallest repeated unit). In crystallography, it is common to define basis vectors so that they are close to a cubic lattice (angles between $\frac{\pi}{3}$ and $\frac{\pi}{2}$ ) but do not necessarily correspond to the primitive unit cell; see Fig. S1 (Supplemental Material [52]). Second, in connection to the lattice, the Minkowski lattice type $G^{6-}, G^{6+}$, or $G^{7+}$ refers to the number and positions of the reference-particle contacts with its neighbors. The Bravais lattice type is not equivalent because it refers to particle centers. We can draw a connection if we consider the space-filling packing of Voronoi polyhedra for the face-centered-cubic (fcc) and the body-centered-cubic (bcc) packings, which are the rhombic dodecahedron and the truncated octahedron, respectively. We can then think of the fcc packing as an example of $G^{6-}$ (with 12 contacts) and the bcc packing as an example of $G^{7+}$ (with 14 contacts).

We note that Betke and Henk [31] developed another method to calculate the densest lattice packings that makes use of Minkowski's result (see point 1 in Sec. II B). Their algorithm systematically checks feasible combinations of vectors on the faces of the difference body of the reference 

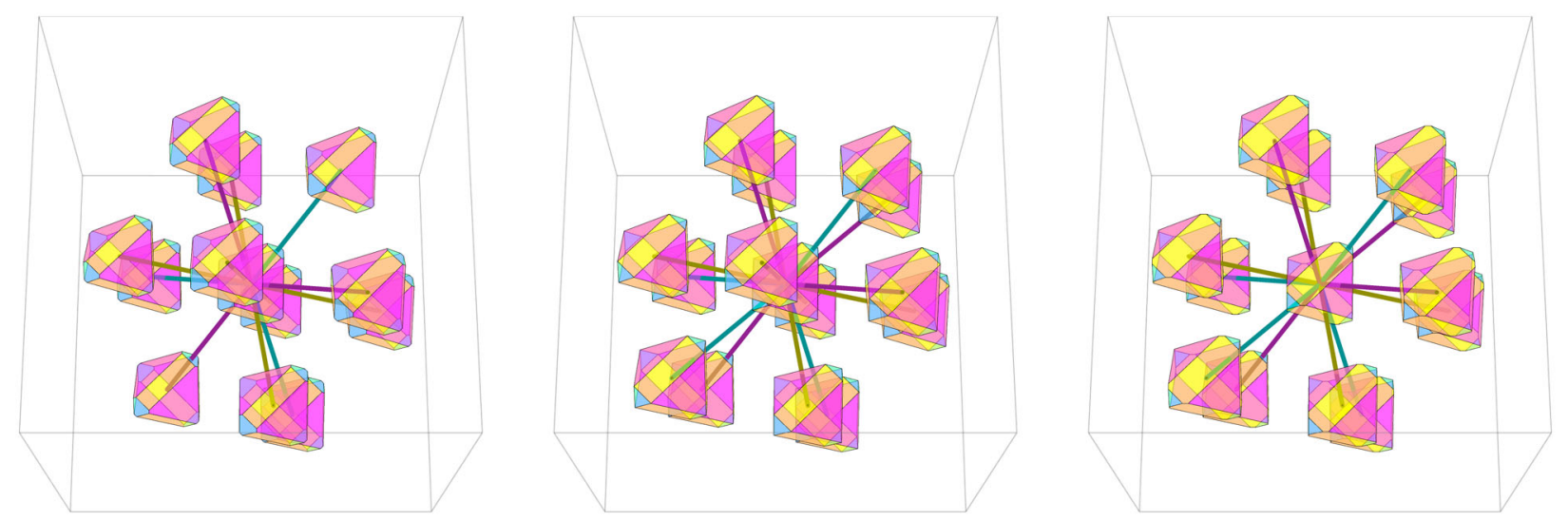

FIG. 2 Example of Minkowski lattice types $G^{6-}, G^{7+}$, and $G^{6+}$ (left to right) for a noncentrally symmetric polyhedron. Lines connect the reference polyhedron at the origin to its neighbors' centers. The lines indicate the color of the faces of the reference polyhedron that touches the neighboring polyhedra. The lines are darker shades of \{magenta, cyan, and yellow $\}$ to aid the eye. The packings correspond to the $323 \cdot 1$-family region $\rho_{4}$, the ridge $\rho_{4} \wedge \rho_{0}$, and the region $\rho_{0}$ (left to right).

shape, where the neighbors' centers live, and is thus deterministic, not probabilistic.

\section{Kuperberg pairs}

A double-lattice packing of a convex particle $\Xi$ has two antiparallel particles in the primitive unit cell $(n=2)+\Xi$ and $\delta-\Xi$ that form a Kuperberg pair [49]; see Fig. 1 (right). The Kuperbergs extended Minkowski's theory to double-lattice packings. They showed that the pair often packs densely for particles without central symmetry and is a candidate for the solution of the general packing problem in situations when lattice packings are not a good solution. Chen et al. [34,51] and Haji-Akbari et al. [38] used Kuperberg pairs in their studies of the densest packings.

Exceptions where the densest packings are realized in neither the Minkowski lattice nor a Kuperberg pair are known. For example, the densest packing of ellipsoids has $n=2$ but is not a double-lattice packing because the two ellipsoids in the primitive unit cell are not antiparallel [41]. The densest packing of tetrahedra requires $n=4$ $[34,35,37,38]$. In two dimensions, space-filling packings (tilings) of pentagons are known with $n=2,3,4,6,8$ [53].

\section{CONSTRUCTION OF TWO-PARAMETER FAMILIES OF POLYHEDRA}

In order to define a packing problem, we need to specify the container and the objects. Here, we focus on packing in infinite Euclidean space, so there is no container. The other parameter we need to specify is the object or particle. Because the maximum packing density is a function of the geometric shape of the particles, it is useful to have an analytical and continuous way to describe shape, i.e., an $N$-parameter family of shapes. An $N$-parameter family of three-dimensional shapes is a function $F: \mathbf{R}^{N} \rightarrow \mathbf{R}^{3}$. The parameters $X=\left\langle X_{1}, \ldots, X_{N}\right\rangle \in \mathbf{R}^{N}$ then represent specific operations on the shape $\Xi=F(X)$. The advantages of such a construction are that (i) it gives us a continuous function of shape, so we can then use as fine a grid as we wish to study packing properties, and (ii) the maximum-density surface that we calculate is also a continuous function of shape and thus easier to manipulate mathematically.

\section{A. Spheric triangle groups}

We introduce families of polyhedra that interpolate between various symmetric solids (Platonic, Archimedean, Catalan) via truncation. The amount of truncation is varied in each family by shifting the truncation planes radially in a manner respecting a centrosymmetric point-symmetry group. Such symmetry groups are known as (finite) spheric triangle groups $\Delta_{p, q, r}$. A spheric triangle group is generated by three reflections across the sides of a spheric triangle with angles $\left\{\frac{\pi}{p}, \frac{\pi}{q}, \frac{\pi}{r}\right\}$. The finite irreducible spheric triangle groups are $\Delta_{3,2,3}$ (tetrahedral, Schönflies notation $T_{d}$ ), $\Delta_{4,2,3}$ (cubic/octahedral, $O_{h}$ ), and $\Delta_{5,2,3}$ (dodecahedral/icosahedral, $I_{h}$ ) [54].

\section{B. Truncation planes}

Three two-parameter families of polyhedra are constructed by truncating the vertices and edges of the dodecahedron or icosahedron (523 family), the cube or octahedron (423 family), and the tetrahedron (323 family). The results are three types of equivalent face-normal vectors $\{\alpha, \beta, \gamma\}$ that are axes of $\{p, q, r\}$-fold symmetry (rotation by angles $\left\{\frac{2 \pi}{p}, \frac{2 \pi}{q}, \frac{2 \pi}{r}\right\}$ ). The triangle group maps any axis to any other axis of the same type. We define the polyhedron $\Xi$ as the intersection of half-spaces for all normal vectors, where the parameters $\{a, b, c\}$ specify the amount of truncation or position of the bounding plane:

$$
\Xi\left\{\begin{array}{l}
\xi \cdot \alpha \leq a \\
\xi \cdot \beta \leq b \\
\xi \cdot \gamma \leq c
\end{array}\right.
$$



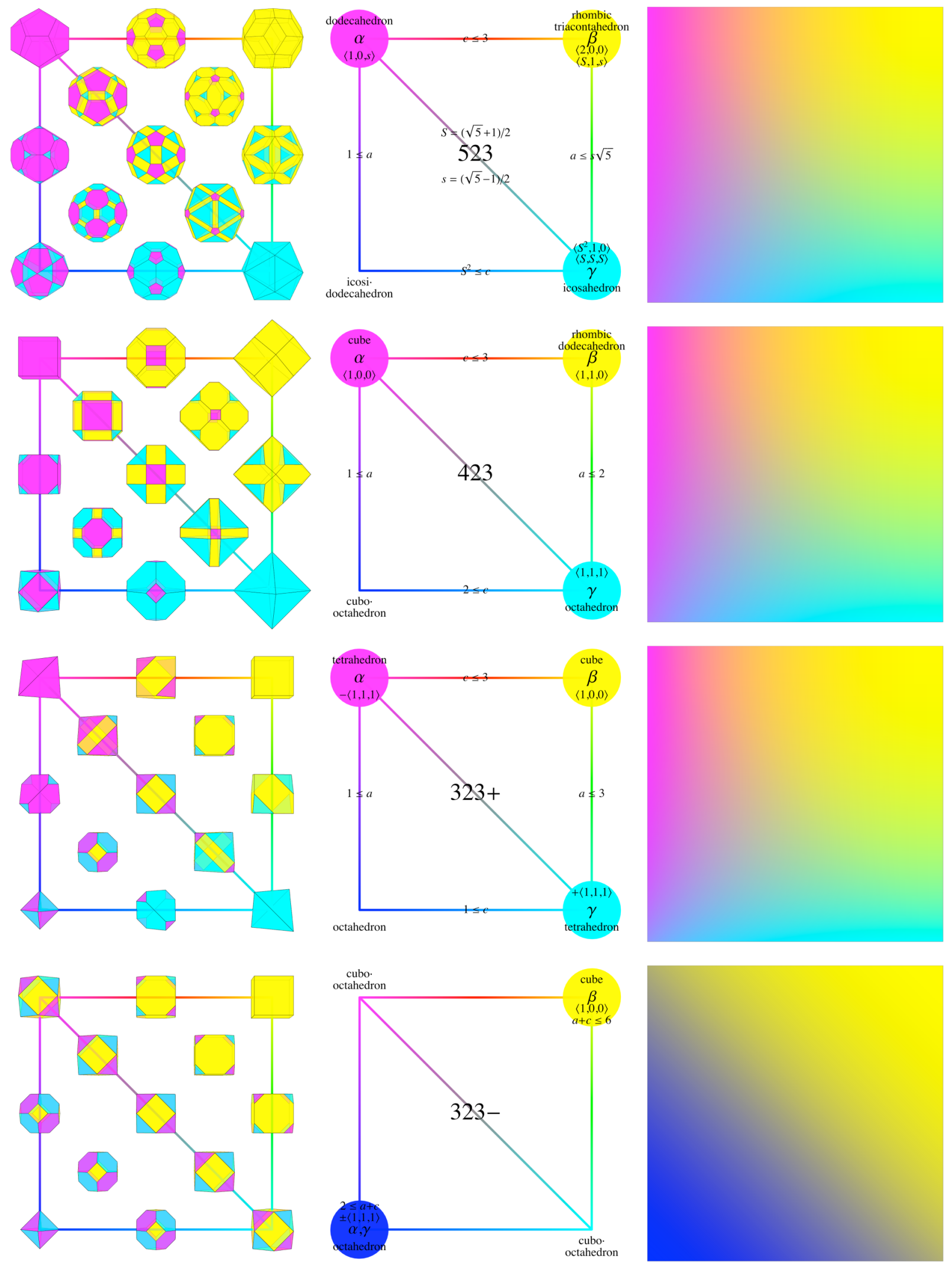

FIG. 3 Left column: Representative polyhedra for the families 523, 423, 323+, and 323-. The two pairs of diagonally opposite corners correspond to dual polyhedra. The 323 family also has reflection symmetry about the diagonal $a=c$. Middle column: The direction of face-normal vectors $\{\alpha, \beta, \gamma\}$, the parameter range $\langle a, c\rangle$, and the names of the corner polyhedra. Right column: A color map of the surface-area fraction of face types. In all columns, the color $\{$ magenta, yellow, and cyan $\}$ indicates the type of face $\{\alpha, \beta, \gamma\}$. 
The normal vectors and parameter ranges for the three families are given by

$$
\begin{aligned}
& 523\left\{\begin{array}{cc}
\alpha=\langle 1,0, s\rangle & 1 \leq a \leq s \sqrt{5} \\
\beta=\langle 2,0,0\rangle & 2=b \\
\gamma=\langle S, S, S\rangle & S^{2} \leq c \leq 3,
\end{array}\right. \\
& S=\frac{1}{2}(\sqrt{5}+1), \quad s=\frac{1}{2}(\sqrt{5}-1), \\
& 423\left\{\begin{array}{cc}
\alpha=\langle 1,0,0\rangle & 1 \leq a \leq 2 \\
\beta=\langle 1,1,0\rangle & 2=b \\
\gamma=\langle 1,1,1\rangle & 2 \leq c \leq 3
\end{array}\right. \\
& 323\left\{\begin{array}{cc}
\alpha=-\langle 1,1,1\rangle & 1 \leq a \leq 3 \\
\beta= \pm\langle 1,0,0\rangle & 1=b \\
\gamma=+\langle 1,1,1\rangle & 1 \leq c \leq 3 .
\end{array}\right.
\end{aligned}
$$

We fix $b$ to be constant because the packing density remains invariant if we rescale all parameters by the same scalar. Figure 3 shows representative polyhedra on the square $\langle a, c\rangle$ parameter domain. Note that at the boundaries of the square domain, the polyhedra have degeneracies (faces with zero area, edges with zero length, coincident points). If we include the degeneracies, all family members have the same number of faces, edges, and vertices. If we exclude the degeneracies, the polyhedra at the corners of the domain are edge transitive. Formulas for the polyhedron volume $U$ and face areas $\{\alpha, \beta, \gamma\}$ are given in the Supplemental Material [52] (p. 5).

\section{Sum and difference bodies}

Most polyhedra in the $p q r$ family have a pointsymmetry group that is identical to the triangle group $\Delta_{p, q, r}$. The only exceptions occur in the 323 family, where the polyhedra with central symmetry are also members of the 423 family, so they have the higher $\Delta_{4,2,3}$ symmetry. In fact, 323 is the only family with noncentrally symmetric polyhedra. We derive two subfamilies: (i) the family of sum bodies $323+$ that is identical to 323 and (ii) the family of difference bodies 323 - that corresponds to 323 along the diagonal $a=c$ and does not change in the orthogonal direction $a+c=0$.

The sum and difference bodies of polyhedra in the 523 and 423 families with central symmetry are identical:

$$
Z=2 \Xi\left\{\begin{array}{l}
\zeta \cdot \alpha \leq 2 a \\
\zeta \cdot \beta \leq 2 b \\
\zeta \cdot \gamma \leq 2 c
\end{array}\right.
$$

For the 323 family without central symmetry, the sum $(323+)$ and difference (323-) bodies are different:

$$
\begin{gathered}
Z=\Xi+\Xi\left\{\begin{array}{l}
\zeta \cdot \alpha \leq a+a \\
\zeta \cdot \beta \leq b+b \\
\zeta \cdot \gamma \leq c+c,
\end{array}\right. \\
Z=\Xi-\Xi\left\{\begin{array}{l}
\zeta \cdot \alpha \leq a+c \\
\zeta \cdot \beta \leq b+b \\
\zeta \cdot \gamma \leq c+a .
\end{array}\right.
\end{gathered}
$$

\section{METHODS}

For each family, we analyze the densest packings in two steps. First, we generate dense packings using Monte Carlo simulations by compressing a small number of $n$ particles with periodic boundary conditions. We then use these results as a guide to construct an analytic surface of maximum packing density (as in Ref. [38] for one shape parameter).

For polyhedra with central symmetry $(523,423$, and 323- families), we investigate only lattice packings because the densest known packings of centrally symmetric shapes are most likely found to be lattice packings [33,36-38]. For polyhedra without central symmetry (323+ family), we study packings with $n=1,2,3,4$ particles in the primitive unit cell. In the following, we use the notation $323 \cdot n$ for a packing of a shape in the 323 family with $n$ particles in the primitive unit cell.

\section{A. Monte Carlo simulated compression}

Our computational techniques closely follow previous works $[37,38,55,56]$. We study small systems of $n$ identical polyhedra in a box of volume $V$ with periodic boundary conditions. The particle positions and orientations evolve in time according to a Monte Carlo trial-move update scheme, where polyhedra are chosen randomly and then rotated and translated by a random amount. In addition, the simulation box is updated in the isobaric-isotension ensemble by randomly perturbing the coordinates of the three box vectors. Strong elongations of the box vectors are avoided by using a lattice-reduction technique. Trial moves are accepted if the generated configurations are free of overlaps and rejected otherwise. Overlap checks are performed using the Gilbert-Johnson-Keerthi (GJK) distance algorithm [57]. In contrast to previous works, here, selfoverlaps are accounted for with periodic copies due to the small dimensions of the simulation box. Compared to other compression techniques in the literature $[33,36,58,59]$, our scheme consistently finds equivalent or denser packings; see Figs. S2 and S3 in the Supplemental Material [52] for a comparison.

For each of the three families, we choose polyhedra from a fine $101 \times 101$-parameter grid in the $\langle a, c\rangle$-parameter domain. In some parts of the domain, where the packings change rapidly with parameters $\langle a, c\rangle$, we apply a finer grid 
to achieve a higher resolution. Although there is some overlap between the shapes of the 423 and 323 families, the total number of unique shapes simulated for this study is more than 55000 . The simulation is initialized at low density and then slowly compressed by gradually increasing the pressure using an exponential protocol over $7 \times 10^{5}$ steps. Because our approach resembles the simulated annealing technique replacing temperature with pressure, we call it "simulated compression." Each compression run is repeated 10 times, and the densest packing is recorded for each parameter choice. The result of the algorithm is a numerical candidate for the densest packing function over the two shape parameters.

\section{B. Analytic optimization}

We use the densest packings from simulated compression as a guide to analytically construct small primitive unit-cell packings that are locally optimal under rotations and translations of the particles.

For a lattice packing $(n=1)$, we perform the following.

(1) We analyze the neighbor contacts in the densest packings obtained with simulated compression.

(2) We write the (abstract) intersection equations in terms of the lattice basis vectors $\{\chi, \psi, \omega\}$ and difference-body faces (where the neighbors' centers live), which are functions of the shape parameters $\langle a, c\rangle$.

(3) We reduce the parameters $\{\chi, \psi, \omega, a, c\}$ to a minimal set of free parameters.

(4) If the (abstract) lattice volume $V=\operatorname{det}[\chi, \psi, \omega]$ has free parameters, we find the values of the free parameters that minimize $V$ and therefore maximize the packing density $\phi=n U / V$.

In general, for a given lattice, there are multiple ways to choose a set of basis vectors that generates the lattice. For centrally symmetric shapes, we choose basis vectors to satisfy one of the three Minkowski types. This choice simplifies the optimization procedure and maintains consistency.

For the double-lattice packings $(n=2)$ in $323 \cdot 2$, we repeat the same process as for the lattice packing with the offset vector $\varsigma$ as an additional parameter and we use the difference body for parallel neighbors and the sum body for antiparallel neighbors. By coincidence, the $n=4$ case (323 . 4 family) reduces to double-lattice packings. The only exception is an area near the tetrahedra $(\langle a, c\rangle=\langle 1,3\rangle$ and $\langle 3,1\rangle)$ that is a double-lattice packing of dimers only slightly rotated (face to face, almost edge to edge). Note that we use a packing of two monomers, whereas the Kuperbergs [49] use a packing of one dimer (Kuperberg pair), and thus there are additional degrees of freedom in our packing. The packing of the $323 \cdot 2$ family (and most of the 323.4 family) is a combination of the sum-body $323+$ and difference-body 323 - packings, in that the neighbors in the densest packing are either parallel or antiparallel.
Because the particles in Kuperberg pairs are related to one another by simple inversion, all intersection equations in the $n=1,2,4$ cases are linear and can be solved analytically. However, for arbitrary shapes (e.g., truncated triangular bipyramids [38]) and $n \geq 3$, rotations between neighboring particles can result in a system of quadratic intersection equations. For example, in the $323 \cdot 3$ family the nonlinear intersection equations must be solved numerically.

The intersection equations of all analyzed families are shown in the Supplemental Material [52] (pp. 6-42). Numeric versus analytic maximum packing densities are compared in the Appendix, Figs. 12-17.

In all cases, we verify that (i) the packing-density data from simulated compression are always a lower bound to the analytic results and (ii) adjacent regions match up correctly.

\section{Classification of packings}

We classify the densest packings based on the types of contacts between neighboring particles in the primitive unit cell. There are six topological types of contact: vertex-vertex, vertex-edge, vertex-face, edge-edge, edgeface, and face-face. Each contact is mathematically expressed in the form of an intersection equation. We refer to packings with the same topological types of contacts and intersection equations as topologically equivalent. Note that the contacts map to each other via isometry between lattice vectors and/or isomorphism between basis vectors. The equivalence relation partitions the domain of each $\mathrm{N}$-parameter family of packings into equivalence classes that we call packing regions and denote by the symbol $\rho_{i}$. Within each region, the packing density varies continuously and smoothly with shape parameters $X$ because the intersection equations and therefore the lattice vectors are smooth (algebraic) functions of the shape parameters $X$. At the boundaries between adjacent regions, the packing density might be nonsmooth (discontinuous derivative). The packing density $\phi(X)$ is necessarily continuous everywhere on the domain.

When crossing between adjacent regions, a minimal set of intersection equations changes. This minimal set depends on the symmetries of adjacent packings and the number of particles in the primitive unit cell. We use the discontinuities of the first derivative of $\phi(X)$ to distinguish three boundary types, as depicted in Fig. 4 . We define the following.

(1) A valley $\left[\phi_{i} \vee \phi_{j}\right]$ is a "soft" boundary. Extending beyond a valley is allowed but gives suboptimal packings. A valley is a generic boundary; intersection equations of adjacent regions need not be related, so the lattice vectors can change discontinuously. We can write the optimal density for a valley as 

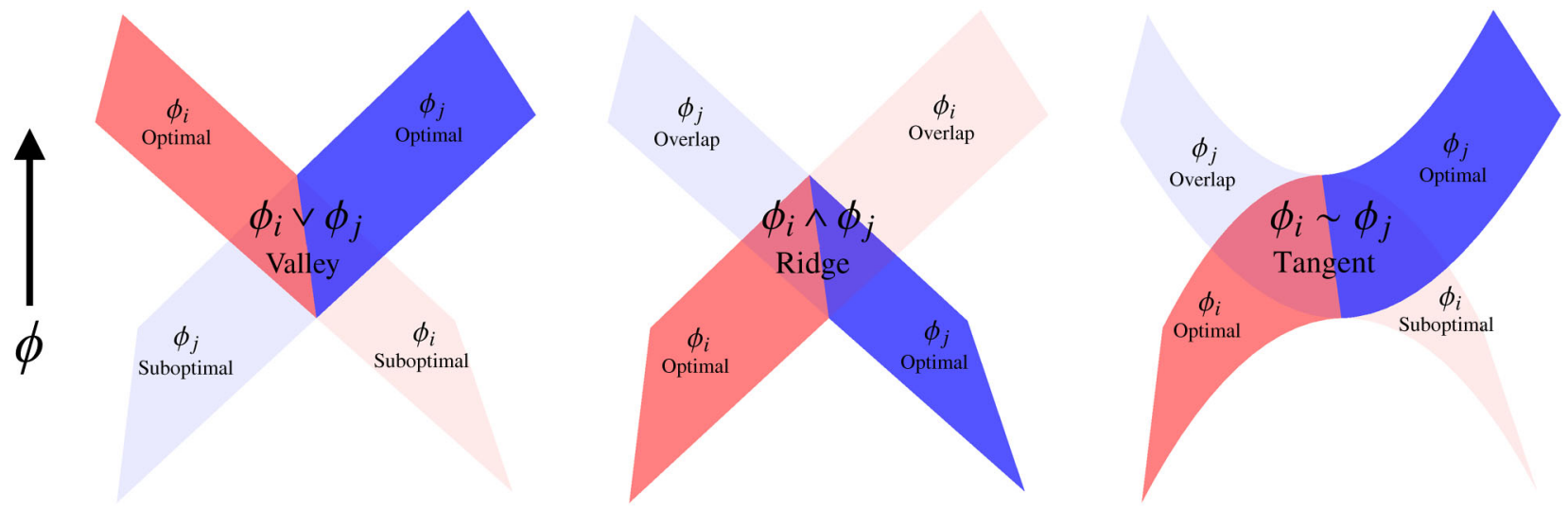

FIG. 4 Boundaries between adjacent regions of topologically distinct packings are classified into three boundary types: valley (left), ridge (middle), and tangent (right).

$$
\phi_{i \vee j}=\left[\phi_{i} \vee \phi_{j}\right]=\max \left\{\phi_{i}, \phi_{j}\right\}= \begin{cases}\phi_{i} & \phi_{i} \geq \phi_{j} \\ \phi_{j} & \phi_{i} \leq \phi_{j}\end{cases}
$$

(2) A ridge $\left[\phi_{i} \wedge \phi_{j}\right]$ is a "hard" boundary. Extending beyond a ridge introduces overlaps into the packing, which is not allowed. The lattice volume and lattice vectors vary continuously but not smoothly across a ridge. On the ridge, the set of contacts is the union of the sets of contacts on both sides. Thus, the set of intersection equations is the union of the sets of intersection equations on both sides. In other words that there are more contacts on the ridge than on either side. We can think of a ridge as a lowerdimensional region in its own right. For a ridge boundary,

$$
\phi_{i \wedge j}=\left[\phi_{i} \wedge \phi_{j}\right]=\min \left\{\phi_{i}, \phi_{j}\right\}= \begin{cases}\phi_{i} & \phi_{i} \leq \phi_{j} \\ \phi_{j} & \phi_{i} \geq \phi_{j}\end{cases}
$$

(3) A tangent $\left[\phi_{i} \sim \phi_{j}\right]\left(\phi_{i} \leq \phi_{j}\right)$ or $\left[\phi_{i} \backsim \phi_{j}\right]\left(\phi_{i} \geq \phi_{j}\right)$ is a hybrid between a valley and a ridge. Extending from the lower-density side to the higherdensity side is allowed but gives suboptimal packings. Extending from the higher-density side to the lower-density side introduces overlaps. The lattice volume and lattice vectors are continuous and smooth across a tangent. The intersection equations of the higher-density region are a subset of the intersection equations of the lower-density region, and in this case, more constraints result in lower packing density. For a tangent boundary, we take partial derivatives in any direction transverse to the tangent boundary, from the $\phi_{i}$ to the $\phi_{j}$ side:

$$
\phi_{i \sim j}=\left[\phi_{i} \sim \phi_{j}\right]= \begin{cases}\phi_{i} & \partial \phi_{i} \geq \partial \phi_{j} \\ \phi_{j} & \partial \phi_{i} \leq \partial \phi_{j}\end{cases}
$$

$$
\phi_{i \backsim j}=\left[\phi_{i} \backsim \phi_{j}\right]= \begin{cases}\phi_{i} & \partial \phi_{i} \leq \partial \phi_{j} \\ \phi_{j} & \partial \phi_{i} \geq \partial \phi_{j}\end{cases}
$$

\section{RESULTS}

The results from simulated compression and analytic optimization are presented in Figs. 5-10. In each figure, the top $3 \times 3$ grid of images shows the surface of maximal packing density $\phi\langle a, c\rangle, \frac{18}{49} \leq \phi \leq 1$, in 3D space $\langle a, c, \phi\rangle$. The normal vector of the surface of maximum packing density maps to the color sphere: white along the north pole ( $\phi$ direction), bright colors along the equator. The central cube has perspective from the $+\phi$ direction. The peripheral cubes are rotated by a zenith angle from the central cube $\left(\frac{\pi}{3}\right.$ from the $+\phi$ direction).

The bottom row shows a top view on the $\langle a, c\rangle$ plane of the packing regions (separated by solid lines). The colors for each region interpolate between the three colors that correspond to the face types in Fig. 3 \{magenta, yellow, and cyan . On the bottom left, if applicable, the numbers indicate the Minkowski lattice type. The colors indicate which types of faces are in contact between parallel neighbors only. On the bottom center, the numbers label the regions in order of area size, 0 being the smallest. The colors indicate the proportion of each type of face over the shape's total surface area, thus aiding visualization of the dominant faces. Finally, if the polyhedra are not centrally symmetric, there is a bottom right panel. The colors indicate which types of faces are in contact between antiparallel neighbors only.

\section{A. 523 results}

Figure 5 shows the surface of maximum packing density for the 523 family. There are 11 regions $\left\{\rho_{i}\right\}$, all with Minkowski lattice type $G^{6-}$, which includes fcc. All boundaries are valleys, so the overall packing-density function is simply the maximum of the functions for each region 


$$
\begin{aligned}
\phi= & \max \left\{\phi_{10}^{6-}, \phi_{9}^{6-}, \phi_{8}^{6-}, \phi_{7}^{6-}, \phi_{6}^{6-}, \phi_{5}^{6-}, \phi_{4}^{6-},\right. \\
& \left.\phi_{3}^{6-}, \phi_{2}^{6-}, \phi_{1}^{6-}, \phi_{0}^{6-}\right\},
\end{aligned}
$$

where we enumerate the regions (subscripts) and indicate the Minkowski lattice type (superscripts).

Consider the colors in the bottom left panel and the numbers in the bottom center. Along the sequence of regions $\left\{\rho_{6}, \rho_{4}, \rho_{5}, \rho_{8}, \rho_{7}, \rho_{2}, \rho_{3}\right\}$, contacts of yellow $\{\beta\}$ faces between neighbors (which are perpendicular to the twofold axes) are gradually replaced by contacts of cyan $\{\gamma\}$ faces (threefold axes). The same is true for the sequence $\left\{\rho_{6}, \rho_{9}, \rho_{10}\right\}$, where contacts of yellow faces are gradually replaced by contacts of magenta $\{\alpha\}$ faces (fivefold axes). There is a close similarity between the transitions in colors characterizing the face contact (bottom left panel) and the colors characterizing the face area (bottom center panel). This similarity in the transition in color demonstrates that the face type with the largest area also has the maximum number of neighbor contacts.

\section{B. 423 results}

Figure 6 shows the surface of maximum packing density for the 423 family. We find 18 regions enumerated in the bottom center panel. Figure 6 shows a complex surface, where seven regions meet at the point $\langle a, c\rangle=\left\langle\frac{6}{5}, \frac{12}{5}\right\rangle$. All boundaries are valleys except for three ridges $\left[\phi_{15}^{6-} \wedge \phi_{11}^{6+}\right]$, $\left[\phi_{13}^{6+} \wedge \phi_{7}^{6-}\right]$, and $\left[\phi_{12}^{6-} \wedge \phi_{3}^{6+}\right]$ and one tangent $\left[\phi_{2}^{7+} \sim \phi_{0}^{6+}\right]$. We can combine regions so that they all intersect at valleys; thus, the overall packing-density function is simply the maximum of the packing-density functions for each region:

$$
\begin{aligned}
\phi= & \max \left\{\phi_{15 \wedge 11}, \phi_{13 \wedge 7}, \phi_{12 \wedge 3}, \phi_{2 \sim 0}, \phi_{17}^{6-}, \phi_{16}^{6-}, \phi_{14}^{7+},\right. \\
& \left.\phi_{10}^{7+}, \phi_{9}^{7+}, \phi_{8}^{7+}, \phi_{6}^{7+}, \phi_{5}^{7+}, \phi_{4}^{7+}, \phi_{1}^{6-}\right\} .
\end{aligned}
$$

Unlike the 523 family, here the colors in the bottom left panel (characterizing the face contact) do not always follow the colors in the bottom center panel (characterizing the face area). The two panels transition in color in the same direction across valleys and in the opposite direction across ridges. An example is the sequence $\left\{\rho_{14}, \rho_{11}, \rho_{15}\right\}$. While the boundary between $\rho_{14}$ and $\rho_{11}$ is a valley and the color changes from cyan toward purple in both panels, $\rho_{15}$ has more cyan than $\rho_{11}$ in the bottom left panel. This change in color means that parts of the magenta faces that were in contact in $\rho_{11}$ are no longer in contact in $\rho_{15}$, despite the magenta faces being larger in $\rho_{15}$.

Furthermore, we observe that at a ridge, the Minkowski lattice type always changes from $G^{6-}$ to $G^{6+}$. We can rationalize this behavior in the following way. By definition, a ridge is the boundary where if we extend beyond from either side, the packing has overlaps. The implication is that as the shape is deformed, a new contact is introduced exactly on the ridge. That new contact will become an overlap if we extend beyond the ridge. There are only three possible types of lattice packings $\left(G^{6-}, G^{6+}, G^{7+}\right)$, and two of them $\left(G^{6-}\right.$ and $\left.G^{6+}\right)$ have the same number of contacts. Thus, the only way that regions can intersect via a ridge is if the packings have type $G^{6-}$ on one side, type $G^{6+}$ on the other side, and type $G^{7+}$ at the boundary. An example of the three Minkowski lattice types across a ridge is shown in Fig. 2.

\section{323 family}

Figure 7 shows the surface of maximum packing density for the 323.1 family. There are eight regions of all three Minkowski lattice types, symmetric about the diagonal $a=c$. All boundaries are valleys except for two ridges $\left[\phi_{6}^{6-} \wedge \phi_{1}^{6+}\right]$ and $\left[\phi_{4}^{6-} \wedge \phi_{0}^{6+}\right]$. By combining regions separated by ridges, we can write down the overall packing-density function simply as the maximum of the packing-density function for each region:

$$
\phi=\max \left\{\phi_{6 \wedge 1}, \phi_{4 \wedge 0}, \phi_{7}^{7+}, \phi_{5}^{7+}, \phi_{3}^{7+}, \phi_{2}^{7+}\right\} .
$$

As required, ridges separate regions of Minkowski lattice types $G^{6-}$ and $G^{6+}$ and have type $G^{7+}$ at the boundary; see Fig. 2.

Figure 8 shows the surface of maximum packing density for the $323 \cdot 3$ family. We have not constructed the analytic surface because there are variable rotations among the three particles in the primitive unit cell that give quadratic (nonlinear) intersection equations. The figure gives an impression of the noise in the raw numerical data and thus demonstrates the significance of the analytic optimization for obtaining a clean surface of maximum packing density. The comparison of the maximum densities for $323 \cdot 1$ and $323 \cdot 3$ shows that three particles in the primitive unit cell pack denser than lattice packings.

Figure 9 shows the surface of maximum packing density for the $323 \cdot 2$ family. The surface is remarkably complex and has reflection symmetry about the diagonal $a=c$. Since all packings are double-lattice packings, we can solve for the intersection equations analytically. There are $8 \times 1+62 \times 2=132$ regions, taking into account reflection symmetry. Eight regions straddle the diagonal, and 62 mirror pairs are reflected across the diagonal. All boundaries are valleys except 13 ridges and 11 tangents. We find multiple junctions where several ridges or several tangents meet at a point.

Figure 10 shows the surface of maximum packing density for the $323 \cdot 4$ family. There are $8 \times 1+61 \times 2=$ 130 regions, eight that straddle the diagonal, and 61 mirror pairs reflected across the diagonal. All boundaries are valleys except 13 ridges and 11 tangents, which are the same as for the $323 \cdot 2$ above.

On the symmetry axis $a=c$, which is identical to the subfamily of 323- polyhedra with central symmetry, all 

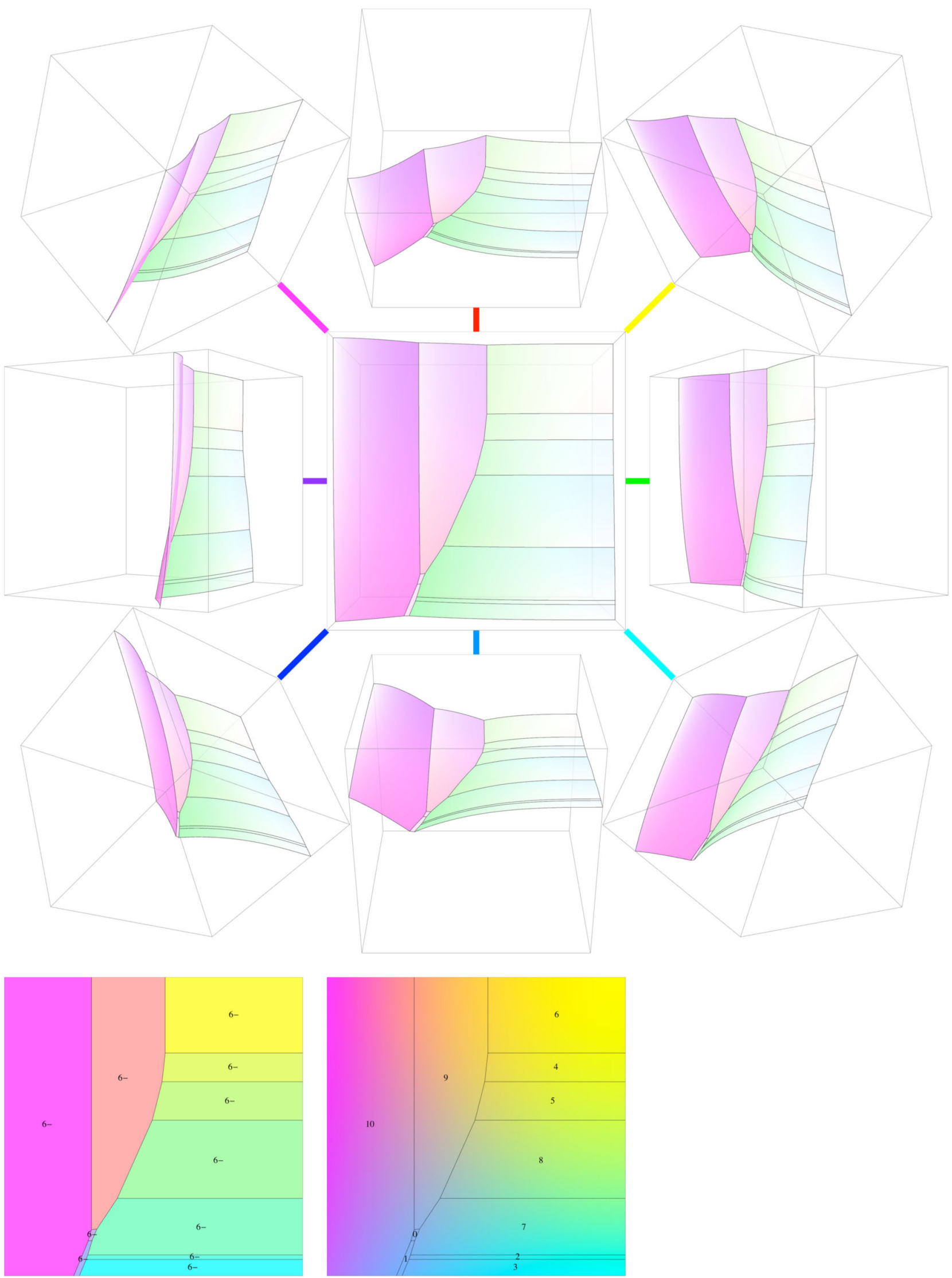

FIG. 5 523-family optimal density surface $(3 \times 3$ grid of cubes), parallel contacts (bottom left), face areas, and region size (bottom center). 

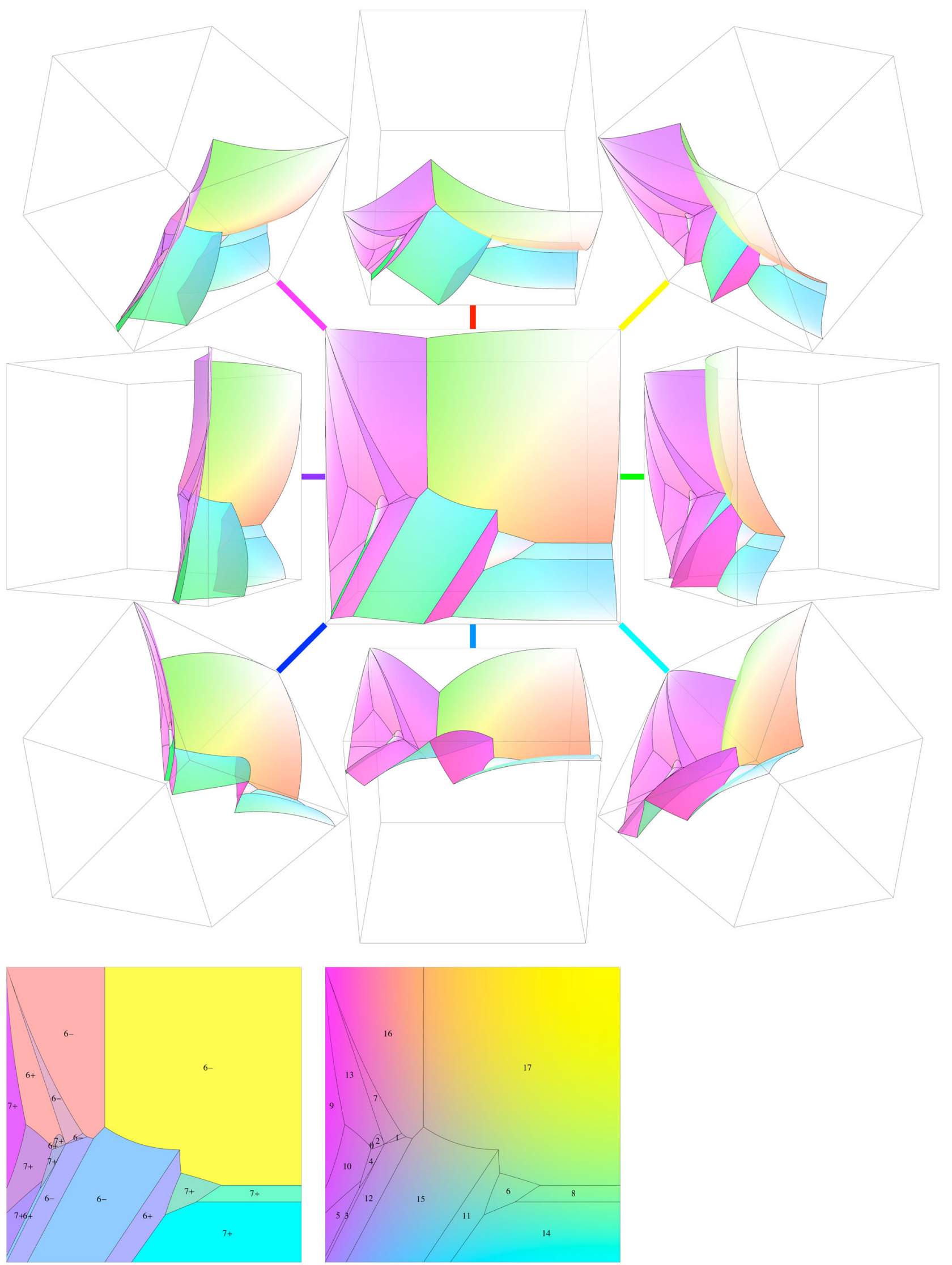

FIG. 6 423-family optimal density surface $(3 \times 3$ grid of cubes), parallel contacts (bottom left), face areas, and region size (bottom center). 

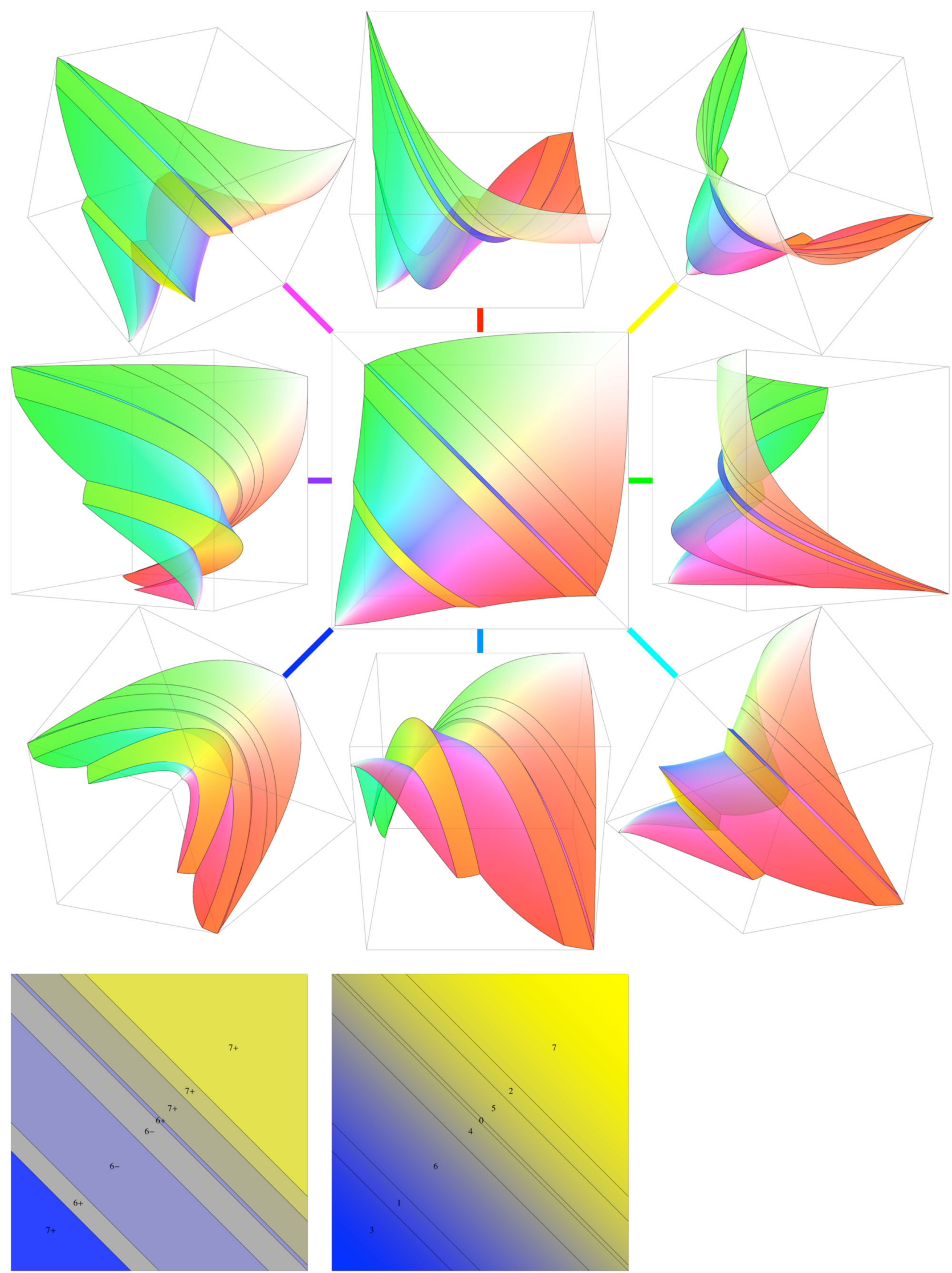

FIG. $7323 \cdot 1$-family optimal density surface $(3 \times 3$ grid of cubes), parallel contacts (bottom left), face areas, and region size (bottom center). 


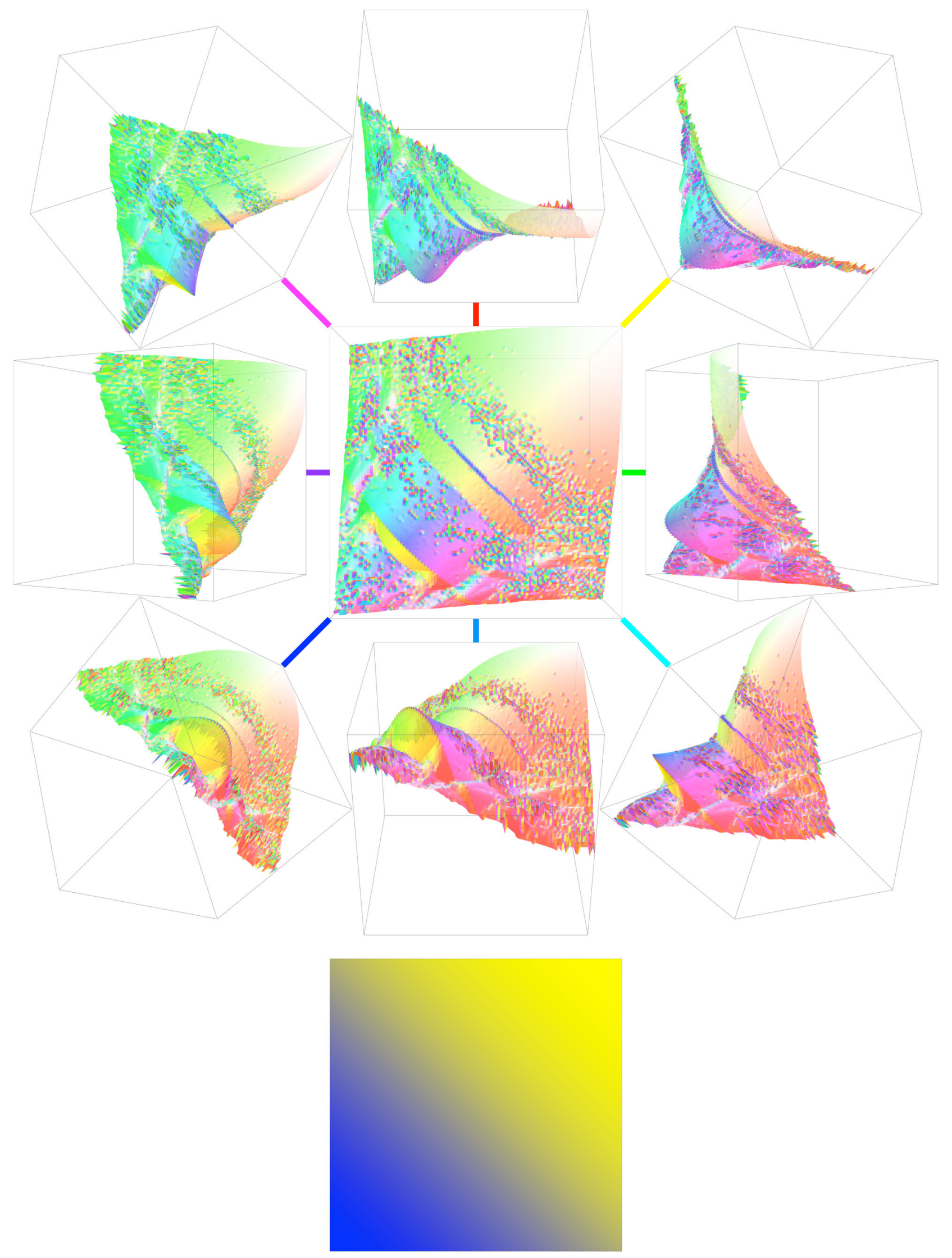

FIG. $8323 \cdot 3$-family optimal density surface $(3 \times 3$ grid of cubes) and face areas (bottom center). 

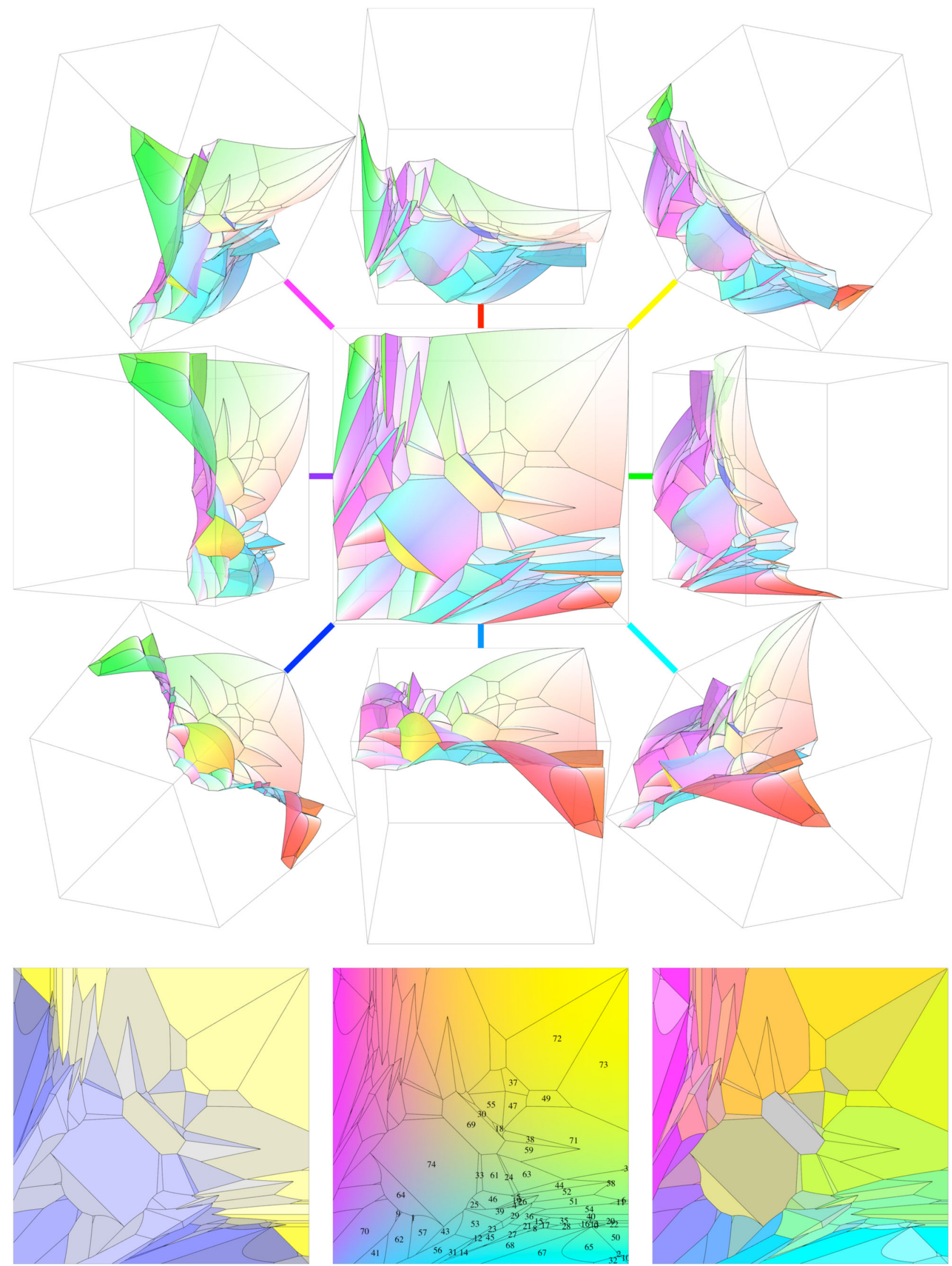

FIG. $9323 \cdot 2$-family optimal density surface $(3 \times 3$ grid of cubes), parallel contacts (bottom left), antiparallel contacts (bottom right), face areas, and region size (bottom center). 

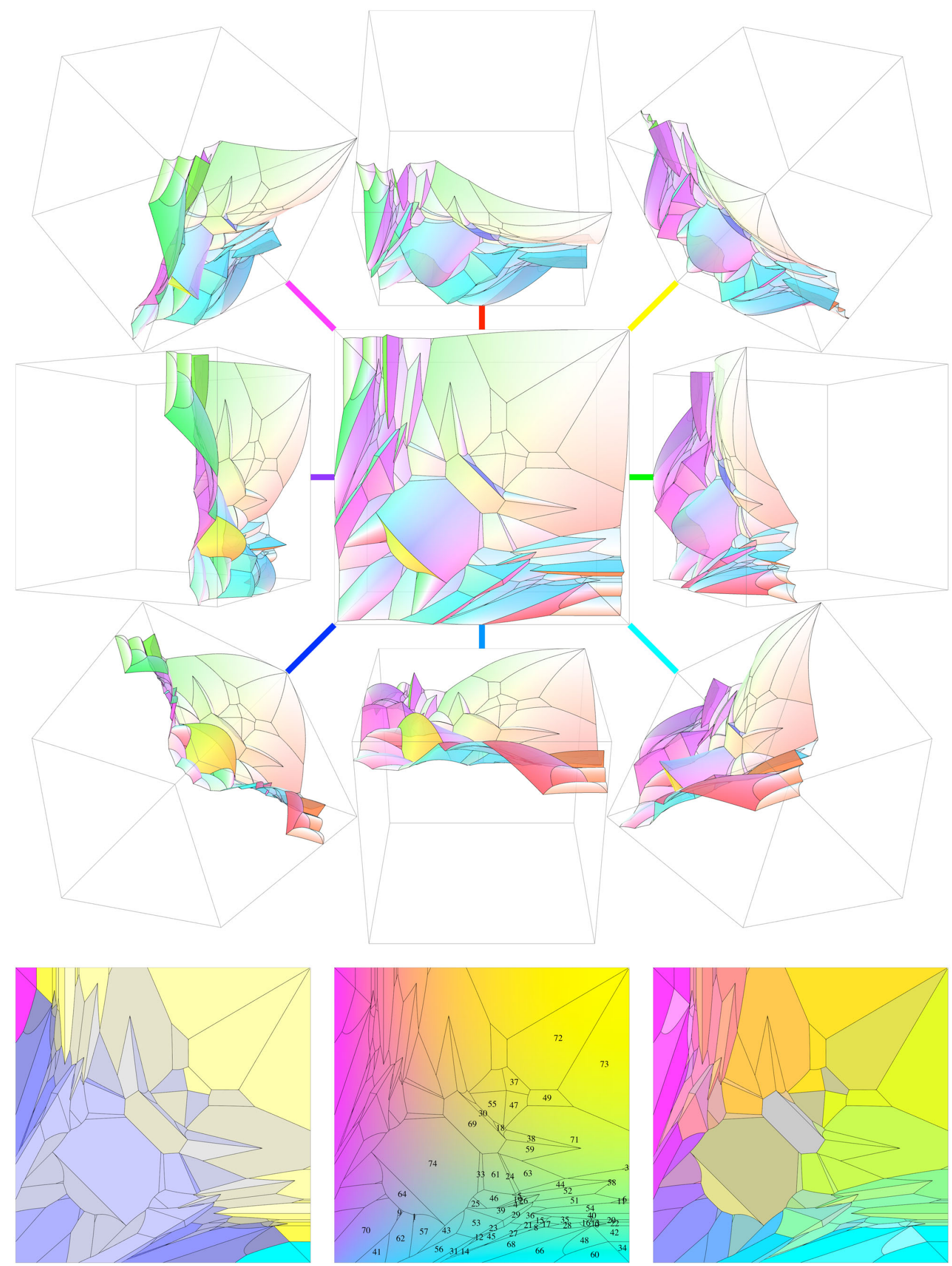

FIG. $10323 \cdot 4$-family optimal density surface $(3 \times 3$ grid of cubes), parallel contacts (bottom left), antiparallel contacts (bottom right), face areas, and region size (bottom center). 
323 families have the same surface of maximum packing density and the same packing, which is the Minkowski lattice $G^{7+}$. Near the symmetry axis, in the regions straddling it, all 323 families have the same density function but possibly different packings. The densest packings for $323 \cdot 2$ and $323 \cdot 4$ are identical except for a small area near the tetrahedron corners $\langle a, c\rangle=\langle 3,1\rangle$ and its mirror image $\langle 1,3\rangle$, where the regions $\rho_{60}$ and $\rho_{34}$ consist of a Kuperberg pair of dimers.

\section{Comparison with other packing studies}

Packing studies of one-parameter families have observed some of the maximum-density surface topography presented here [33,36-38,41-43,46]. In this section, we apply our classification of regions of packings and boundaries to earlier works and compare their analyses of the maximum-density graphs to our surfaces of maximum density.

In Fig. 3 of Ref. [43] on puffy tetrahedra, Kallus and Elser describe four regions and three transitions, two of them as abrupt (between $D_{1}$ and $S_{1}$, and $S_{1}$ and $D_{0}$ ) and one as continuous (between $S_{0}$ and $D_{1}$ ). From their data, we would define five regions and four transitions (two ridges and two valleys). Specifically, there is a ridge between $S_{0}$ and $D_{1}$, where we expect the lattice vectors to change continuously but not smoothly. A second local maximum within the $S_{1}$ region is not identified as a transition but according to our theory is a ridge, which means the two sides must have different intersection equations.

The one-parameter family of truncated tetrahedra in Ref. [37] [see their Fig. 2(a)] corresponds to the left edge (and by symmetry also to the bottom edge) of the 323.4 family. The authors find eight regions and seven transitions, with which we are in agreement: $\left\{\rho_{60}, \rho_{66}, \rho_{68}, \rho_{31}, \rho_{56}, \rho_{62}\right.$, $\left.\rho_{41}, \rho_{70}\right\}$. We note that their vector-length curves are continuous across ridges, as expected. The ridges are $\left[\rho_{66} \wedge \rho_{68}\right]$ and $\left[\rho_{31} \wedge \rho_{56}\right]$.

The one-parameter family of Gantapara et al. [46] interpolating from the cube to the octahedron via the cuboctahedron corresponds to the symmetry diagonal in the 323 family. While they report 14 regions (see their Fig. 1 and Supplemental Material [52]), we find that some of these regions are identical and instead we identify only eight: $\left\{\rho_{7}, \rho_{2}, \rho_{5}, \rho_{0}, \rho_{4}, \rho_{6}, \rho_{1}, \rho_{3}\right\}$; see Fig. 7. Our region $\rho_{4}$ corresponds to their regions VI, VII, and VIII, and our region $\rho_{6}$ corresponds to their IX, X, XI, and XII. The discontinuities in the lattice basis-vector lengths and angles that appear due to ordering them by magnitude were interpreted by the authors as the boundary X-XI. Other discontinuities were interpreted as boundaries VI-VII, VII-VIII, IX-X, and XI-XII, but we think they appear due to the specific choice of basis vectors. In addition, our region $\rho_{2}$ corresponds to their regions II and III, but we find no boundary in between.

\section{CONCLUSIONS}

We studied three two-parameter families of symmetric polyhedra, where the shape is continuously deformed via vertex and edge truncations. The surface of maximum packing density was determined as a function of shape parameters $\langle a, c\rangle$. We defined an equivalence relation of packings based on the topological type of contacts and intersection equations, which allowed a classification of packings into regions, as well as a definition of three types of boundaries between adjacent regions: valleys, ridges, and tangents. We note that ridges are special boundaries; the lattice deforms continuously across a ridge, but the set of neighbors in contact changes. We have shown that for centrally symmetric shapes, a ridge always separates a region of Minkowski type $G^{6-}$ from a region of Minkowski type $G^{6+}$. On the ridge, the packing has Minkowski type $G^{7+}$.

A similar study of maximum-density surfaces can be performed for general shapes (not necessarily polyhedra). If we define a continuous family of shapes, then the maximum-density surface will also be continuous. Thus, we expect the same classification of boundaries between packing regions (valleys, ridges, and tangents) for general shapes, too. For example, the smoothness of the shape itself (e.g., curvature versus sharp vertices or edges) does not affect our boundary classification as long as the shape parameter varies continuously.

The holy grail of packing studies is to predict structures based solely on shape. It is tempting from the large data set presented here to hope that a simple relation between shape and packing might be discerned. However, our results demonstrate that a complete appreciation of the way polyhedra pack at high density must be achieved through exploration of not only the specific shape of immediate interest but also shapes near it in shape space, as achieved by small deformations. Here, we investigated deformation via truncations, but other shape-anisotropy dimensions may be explored in the same manner [60-62]. This knowledge should be especially helpful in the design and synthesis of particles intended to pack into target structures and/or densities, as we demonstrate below.

Our results suggest the following general principles relating packing and shape that we hope will both serve as useful guidelines to experiments and be tested by theorists.

(i) A single shape descriptor is generally insufficient for predicting the densest packing fractions or structures. Instead, one needs to consider higher-dimensional surfaces of optimal packing densities and/or structures of nearby shapes (in shape space) to anticipate how well or poorly a given shape might pack.

(ii) In experiments, particles at all scales (nano, colloidal, and even 3D printed) have shape imperfections and some degree of polydispersity. The densest packings are more likely to be achieved experimentally and 
more tolerant to small differences if the packing lies in a smooth region of the maximum-density surface. In contrast, it may be very difficult to achieve and less tolerant to imperfections if the packing lies in a complex region of the surface (i.e., adjacent to many different packing regions or on boundaries). For example, for the truncated cube (Fig. 6, region 17), we foresee that small imperfections in the shape will make little difference in the packing structure. On the other hand, for the edge-and-vertex truncated tetrahedron (Fig. 10, region 35), small imperfections in the particle shape may give completely different packing structures than expected.

(iii) The topography of the maximum-density surface as a function of shape is useful if we are interested in obtaining a target density. If the slope of the region is steep, small imperfections in shape will achieve very different packing densities. Knowledge of the underlying surface can help to anticipate how sensitive the packing density is to small shape imperfections and even suggest alternative shapes that yield the desired packing fraction.

(iv) Given the landscape topography in the neighborhood of a specific shape, one can deduce $a$ posteriori what kind of imperfection the sample of shapes may have, in the absence of other information. For example, consider a shape that lies on a region of the landscape where the packing density changes rapidly with edge truncations but not much with vertex truncations, as in region 10 of Fig. 5. The deformation from the dodecahedron to the icosidodecahedron (going down along the left edge) changes the packing density only slightly. We can thus be certain that imperfections on the shape's vertices will make little difference to the packing density. However, if we observe that the packing density in an experiment is much lower than expected for a given perfect shape, we may deduce that our polyhedron has imperfections on its edges, rather than its vertices. We expect such information to be useful in the synthesis of nanoparticles for target applications that exploit dense packings.

(v) For centrally symmetric shapes, we showed that if the shape lies on a surface that is near a ridge, the contacts on either side of the ridge are different, while the lattices are the same. For example, in Fig. 6 going from the octahedron to the cuboctahedron via vertex truncations (moving to the left along the lower edge), there is a ridge between regions 11 and 15; thus, although the contacts are different, the lattice is the same. Furthermore, this feature might allow the fabrication of reconfigurable structures whose interparticle orientations (contacts) can be toggled back and forth while the crystal superlattice remains the same.

An important caveat in the general principles outlined above is that they hold for the two shape parameters explored here. Of course, there are many other shape parameters that may similarly affect the densest packing landscape. Thus, a surface that appears smooth for the two parameters considered here may be rough when other parameters are also considered. Moreover, here we have not considered surfaces of suboptimal packings, which may affect the practical achievability of a given densest packing. These two points further emphasize the value in thinking of shape not as a fixed and static property but rather as part of a continuum of shape-anisotropy characteristics (dimensions). Thus, if one is interested in dense packings, the relevant question to ask is not "what is the densest packing of a particular shape" but rather "what is the maximum-packing-density surface in the neighborhood of that shape?"

\section{ACKNOWLEDGMENTS}

E. R. C. acknowledges the National Science Foundation MSPRF Grant No. DMS-1204686. D. K. acknowledges the FP7 Marie Curie Actions of the European Commission, Grant Agreement No. PIOF-GA-2011-302490 Actsa. The numerical studies of the densest packings were supported by the U.S. Department of Energy, Office of Basic Energy Sciences, Division of Materials Sciences and Engineering, Biomolecular Materials Program, under Award No. DEFG02-02ER46000. S. C. G., P. F. D., and M.E. were supported by the DOD/ASD(R\&E) under Grant No. N00244-09-1-0062. Any opinions, findings, and conclusions or recommendations expressed in this publication are those of the authors and do not necessarily reflect the views of the DOD/ASD(R\&E). E. R.C. and D. K. contributed equally to this work. 


\section{APPENDIX}

$$
\begin{aligned}
& \begin{array}{llll} 
& & & \xi \cdot \alpha \leq a+c \\
\boldsymbol{\sigma}+\boldsymbol{\Xi} \cdot \beta=b+b & \boldsymbol{Z}=\boldsymbol{\Xi}-\boldsymbol{\Xi} & \xi \cdot \beta \leq b+b
\end{array} \\
& \begin{array}{l}
\xi \cdot \beta \leq b+b \\
\xi \cdot \gamma \leq c+a
\end{array} \\
& \Xi \cap \varsigma+\Xi \\
& \varsigma \cdot \alpha=a+c \\
& \varsigma \cdot \gamma=c+a
\end{aligned}
$$
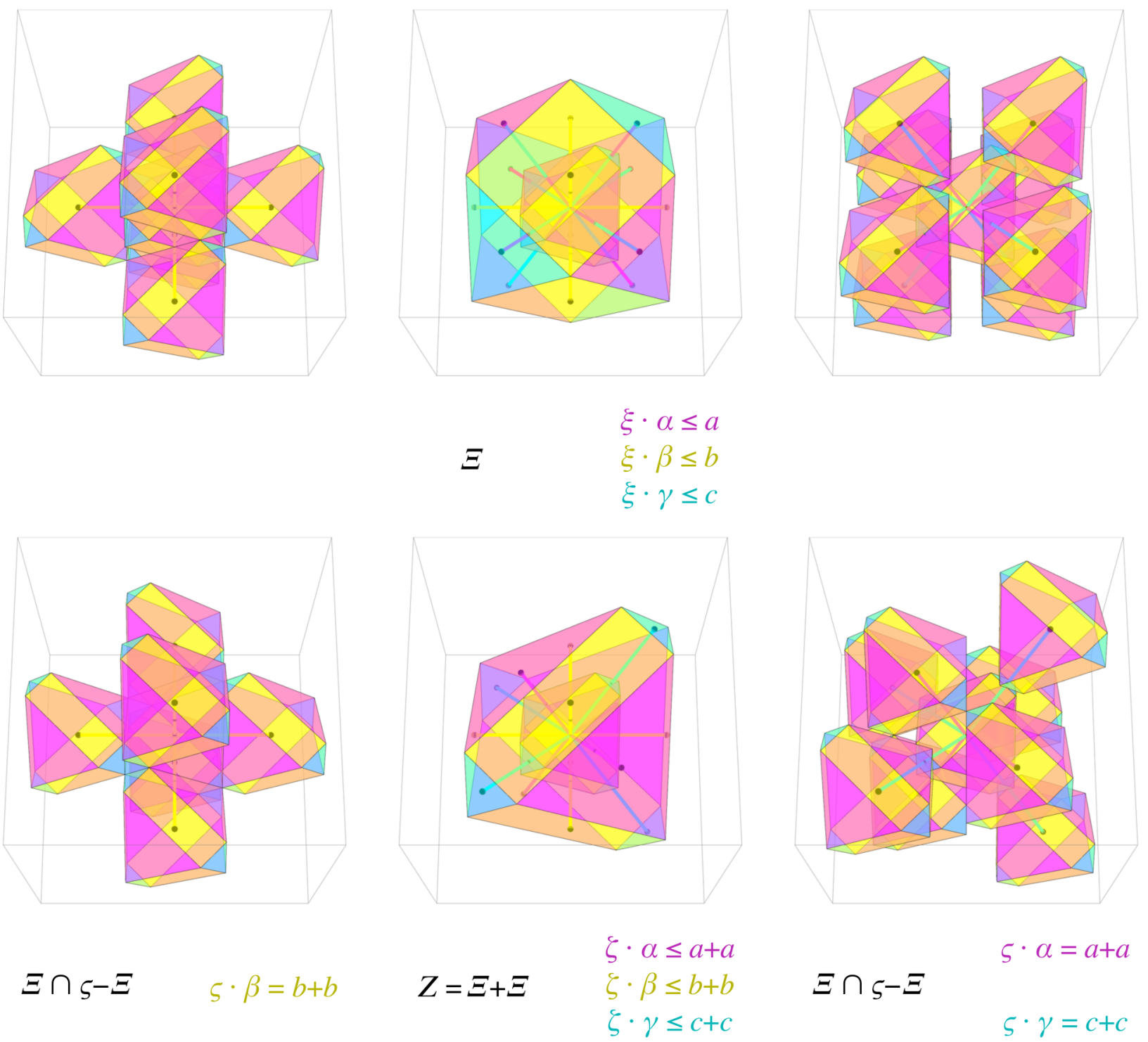

FIG. 11 Sum body 323+ (top center) and difference body 323- (bottom center). 323-family face-face contacts: yellow to yellow between antiparallel neighbors (top left), magenta to magenta and cyan to cyan between antiparallel neighbors (top right), yellow to yellow between parallel neighbors (bottom left), and magenta to cyan and cyan to magenta between parallel neighbors (bottom right). 

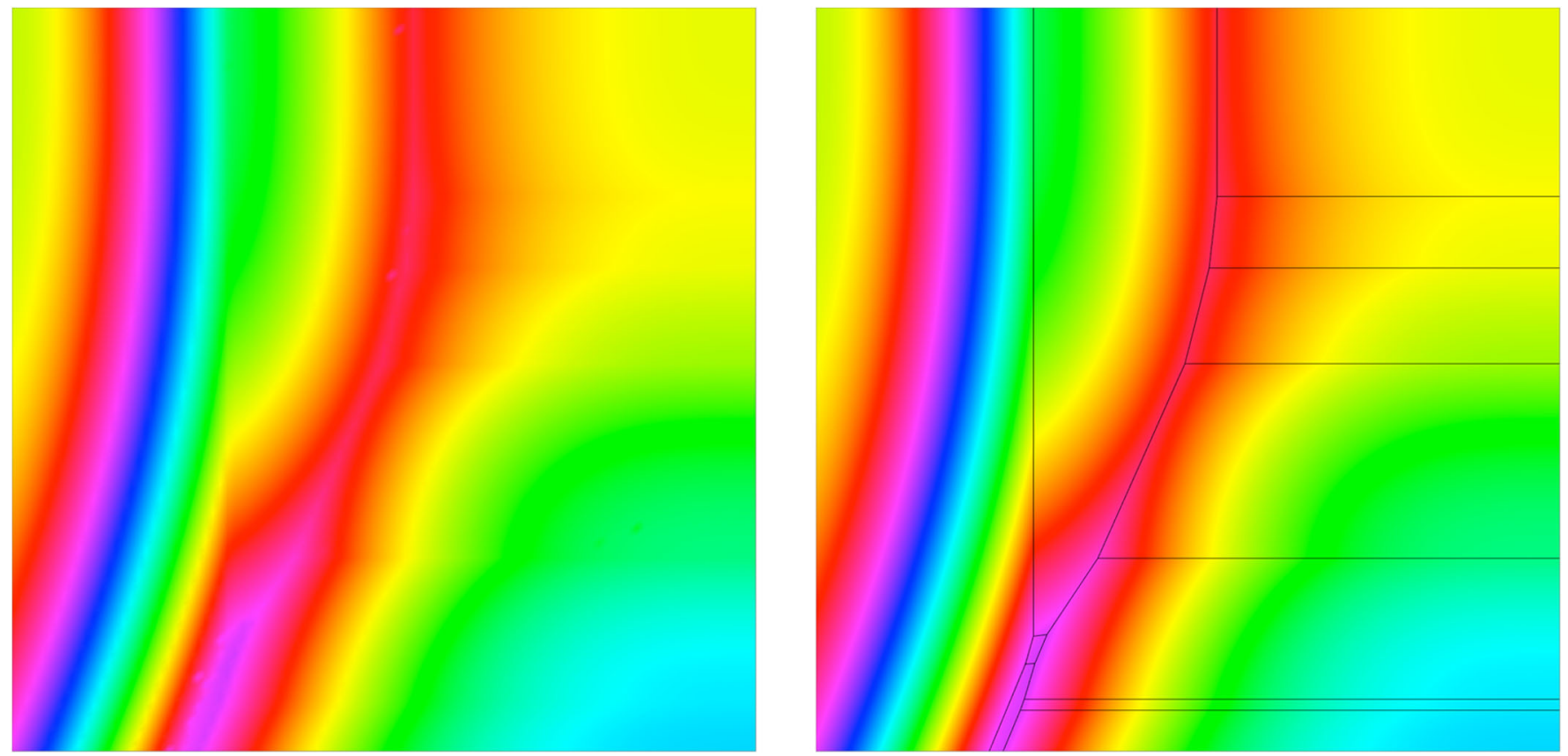

FIG. 12 Contours of optimal packing density $\phi$ for the 523 family, numeric (left) versus analytic (right). The color gradient $\{$ blue, magenta, red, yellow, green, cyan, and blue $\}$ represents the $\phi$ gradient $\left\{\phi-\frac{3}{60}, \phi-\frac{2}{60}, \phi-\frac{1}{60}, \phi, \phi+\frac{1}{60}\right.$, $\left.\phi+\frac{2}{60}, \phi+\frac{3}{60}\right\} ;$ yellow contours occur at $\phi=\left\{\frac{8}{10}, \frac{9}{10}\right\}$.
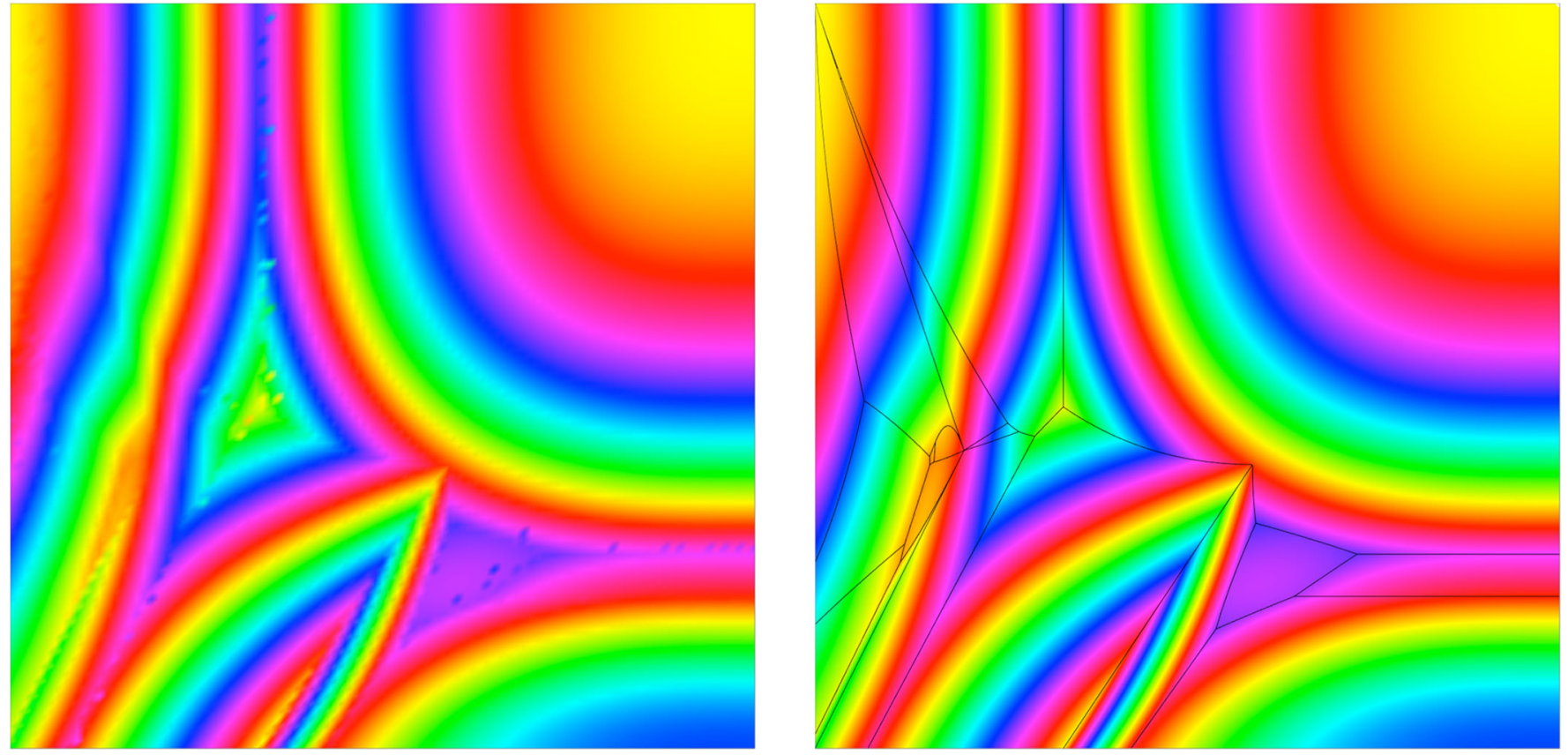

FIG. 13 Contours of optimal packing density $\phi$ for the 423 family, numeric (left) versus analytic (right). The color gradient $\{$ blue, magenta, red, yellow, green, cyan, and blue $\}$ represents the $\phi$ gradient $\left\{\phi-\frac{3}{60}, \phi-\frac{2}{60}, \phi-\frac{1}{60}, \phi, \phi+\frac{1}{60}\right.$, $\left.\phi+\frac{2}{60}, \phi+\frac{3}{60}\right\}$; yellow contours occur at $\phi=\left\{\frac{8}{10}, \frac{9}{10}, 1\right\}$. 

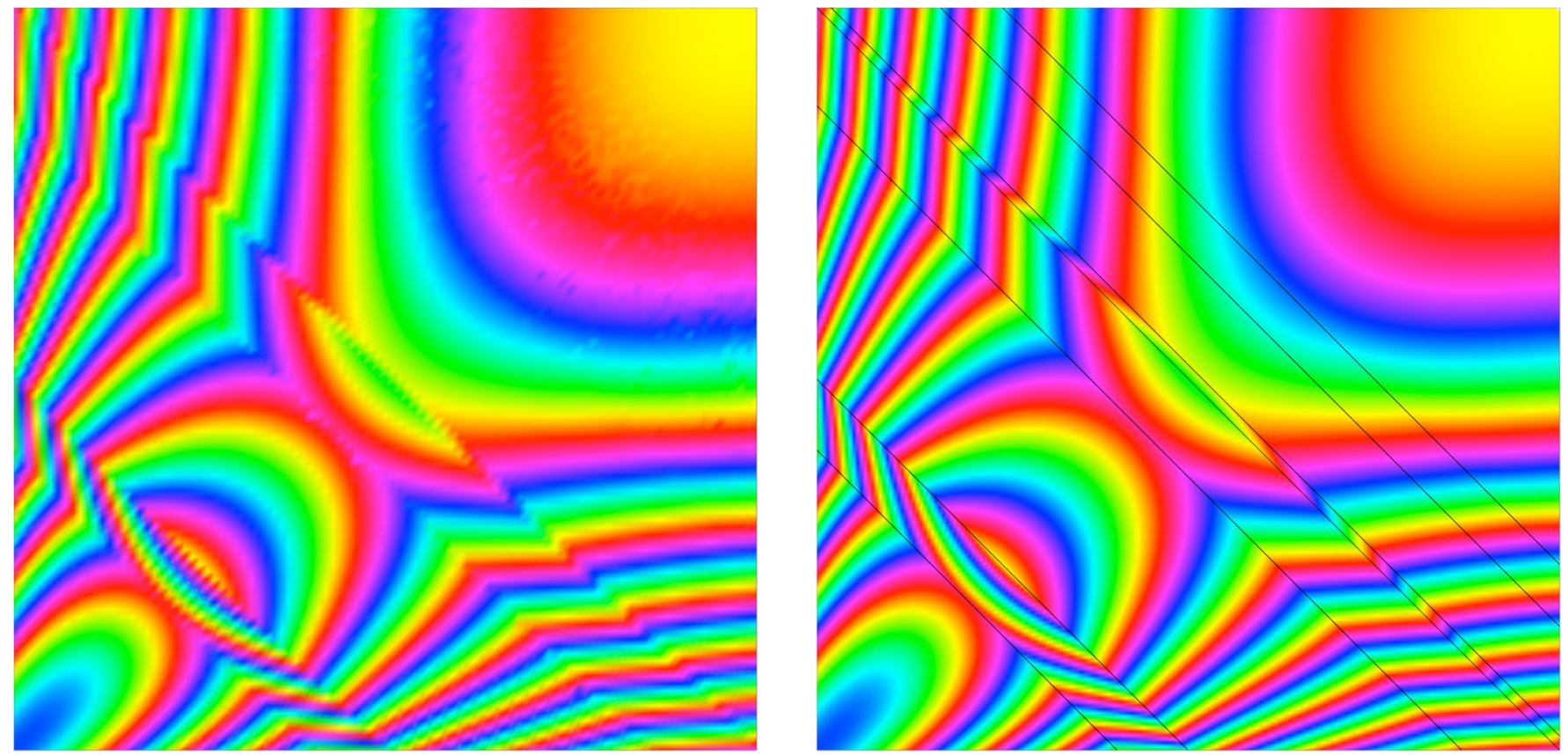

FIG. 14 Contours of optimal packing density $\phi$ for the $323 \cdot 1$ family, numeric (left) versus analytic (right). The color gradient $\{$ blue, magenta, red, yellow, green, cyan, and blue $\}$ represents the $\phi$ gradient $\left\{\phi-\frac{3}{60}, \phi-\frac{2}{60}, \phi-\frac{1}{60}, \phi, \phi+\frac{1}{60}, \phi+\frac{2}{60}\right.$, $\left.\phi+\frac{3}{60}\right\}$; yellow contours occur at $\phi=\left\{\frac{4}{10}, \frac{5}{10}, \frac{6}{10}, \frac{7}{10}, \frac{8}{10}, \frac{9}{10}, 1\right\}$.
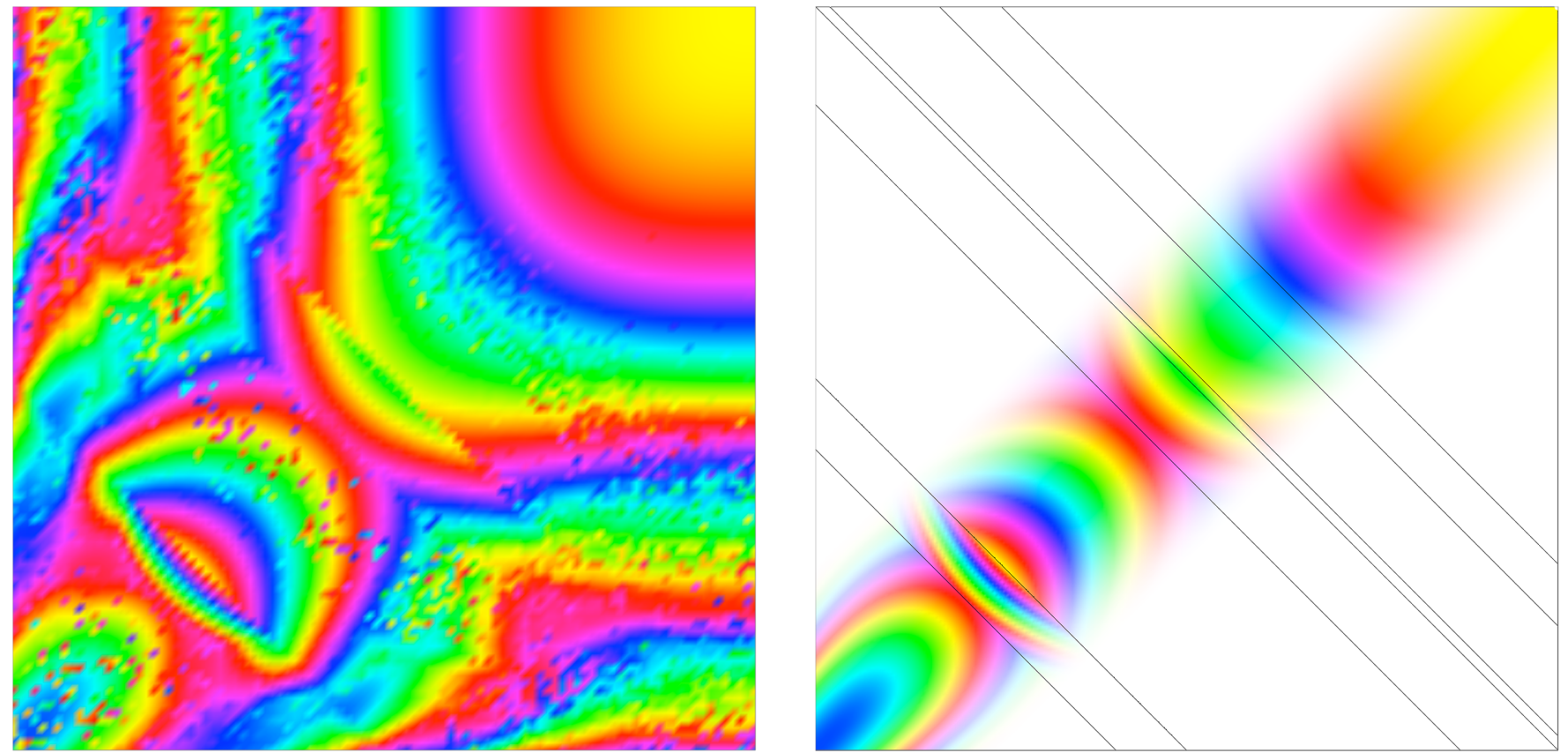

FIG. 15 Contours of optimal packing density $\phi$ for the $323 \cdot 3$ family, numeric (left) versus analytic along the symmetry diagonal (right). The

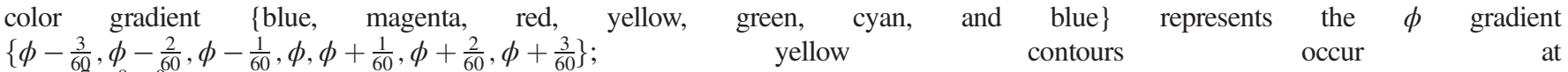
$\phi=\left\{\frac{7}{10}, \frac{8}{10}, \frac{9}{10}, 1\right\}$. 

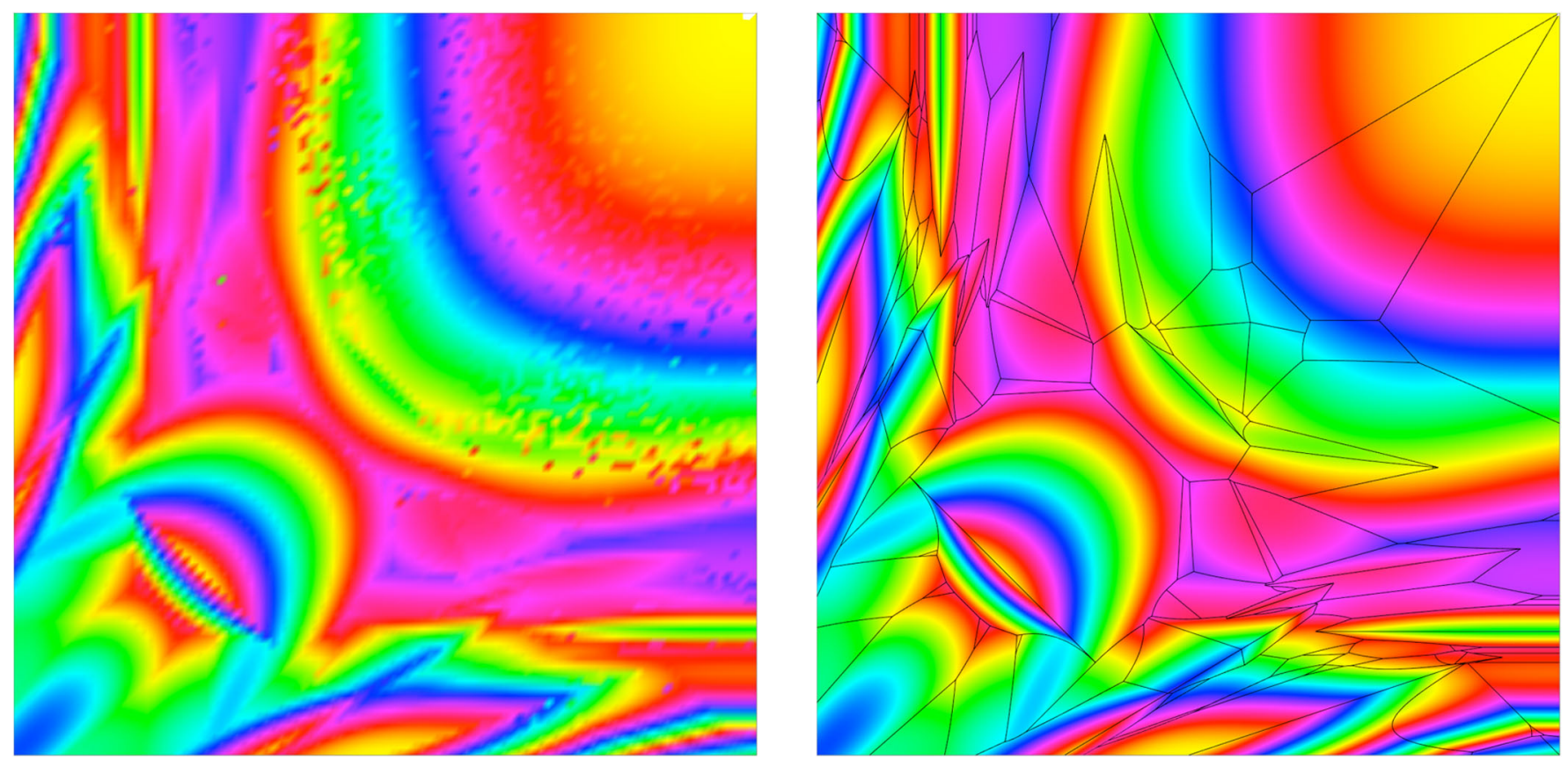

FIG. 16 Contours of optimal packing density $\phi$ for the $323 \cdot 2$ family, numeric (left) versus analytic (right). The color gradient $\{$ blue, magenta, red, yellow, green, cyan, and blue $\}$ represents the $\phi$ gradient $\left\{\phi-\frac{3}{60}, \phi-\frac{2}{60}, \phi-\frac{1}{60}, \phi, \phi+\frac{1}{60}, \phi+\frac{2}{60}\right.$, $\left.\phi+\frac{3}{60}\right\}$; yellow contours occur at $\phi=\left\{\frac{8}{10}, \frac{9}{10}, 1\right\}$.
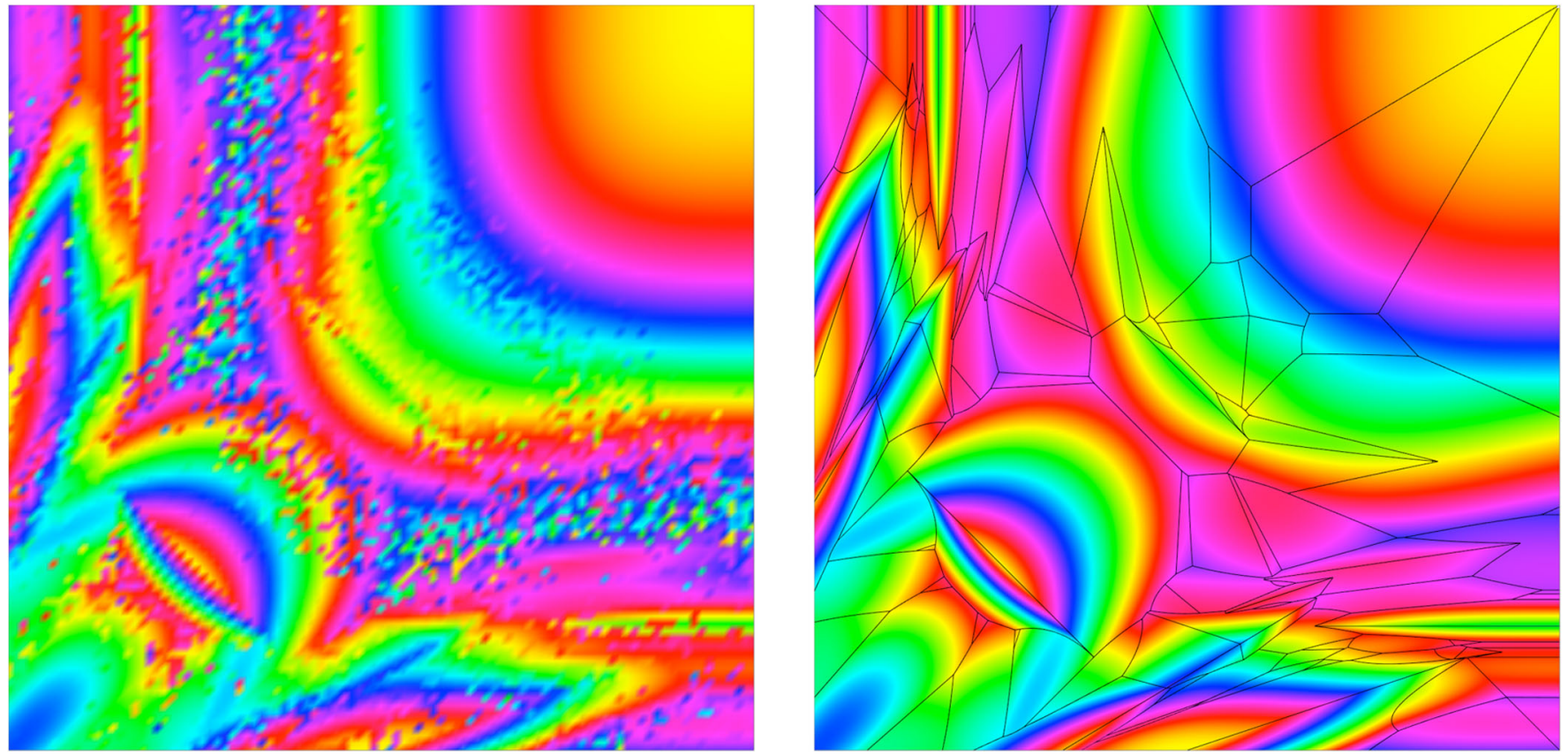

FIG. 17 Contours of optimal packing density $\phi$ for the 323.4 family, numeric (left) versus analytic (right). The color gradient $\{$ blue, magenta, red, yellow, green, cyan, and blue $\}$ represents the $\phi$ gradient $\left\{\phi-\frac{3}{60}, \phi-\frac{2}{60}, \phi-\frac{1}{60}, \phi, \phi+\frac{1}{60}\right.$, $\left.\phi+\frac{2}{60}, \phi+\frac{3}{60}\right\}$; yellow contours occur at $\phi=\left\{\frac{9}{10}, 1\right\}$. 
[1] T. C. Hales, Historical Overview of the Kepler Conjecture, Discrete Comput. Geom. 36, 5 (2006).

[2] A. Bezdek and W. Kuperberg, Dense Packing of Space with Various Convex Solids, arXiv:1008.2398.

[3] T. Aste and D. Weaire, The Pursuit of Perfect Packing (Institute of Physics, Bristol, PA, 2000).

[4] E. D. Demaine and M. L. Demaine, Jigsaw Puzzles, Edge Matching, and Polyomino Packing: Connections and Complexity, Graphs Combinator. 23, 195 (2007).

[5] U. Agarwal and F. A. Escobedo, Mesophase Behavior of Polyhedral Particles, Nat. Mater. 10, 230 (2011).

[6] N. Arkus, V. N. Manoharan, and Michael P. Brenner, Deriving Finite Sphere Packings, SIAM J. Discrete Math. 25, 1860 (2011).

[7] J. Henzie, M. Grünwald, A. Widmer-Cooper, P. L. Geissler, and P. Yang, Self-Assembly of Uniform Polyhedral Silver Nanocrystals into Densest Packings and Exotic Superlattices, Nat. Mater. 11, 131 (2011).

[8] P. Song, B. K. Olmsted, P. Chaikin, and M. D. Ward, Crystallization of Micrometer-Sized Particles with Molecular Contours, Langmuir 29, 13686 (2013).

[9] J.-W. Yoo and S. Mitragotri, Polymer Particles That Switch Shape in Response to a Stimulus, Proc. Natl. Acad. Sci. U.S.A. 107, 11205 (2010).

[10] M. M. Maye, M. T. Kumara, D. Nykypanchuk, W. B. Sherman, and O. Gang, Switching Binary States of Nanoparticle Superlattices and Dimer Clusters by DNA Strands, Nat. Nanotechnol. 5, 116 (2009).

[11] T. D. Nguyen and S. C. Glotzer, Reconfigurable Assemblies of Shape-Changing Nanorods, ACS Nano 4, 2585 (2010).

[12] T. D. Nguyen, E. Jankowski, and S.C. Glotzer, SelfAssembly and Reconfigurability of Shape-Shifting Particles, ACS Nano 5, 8892 (2011).

[13] Y. Zhang, F. Lu, D. van der Lelie, and O. Gang, Continuous Phase Transformation in Nanocube Assemblies, Phys. Rev. Lett. 107, 135701 (2011).

[14] O. Gang and Y. Zhang, Shaping Phases by Phasing Shapes, ACS Nano 5, 8459 (2011).

[15] K. J. Leea, J. Yoon, S. Rahmani, S. Hwang, S. Bhaskar, S. Mitragotri, and J. Lahann, Spontaneous Shape Reconfigurations in Multicompartmental Microcylinders, Proc. Natl. Acad. Sci. U.S.A. 109, 16057 (2012).

[16] K. L. Kohlstedt and S. C. Glotzer, Polymer Particles That Switch Shape in Response to a Stimulus, Phys. Rev. E 87, 032305 (2013).

[17] R. Guo, Z. Liu, X.-M. Xie, and L.-T. Yan, Harnessing Dynamic Covalent Bonds in Patchy Nanoparticles: Creating Shape-Shifting Building Blocks for Rational and Responsive Self-Assembly, J. Phys. Chem. Lett. 4, 1221 (2013).

[18] A. J. Liu and S. R. Nagel, The Jamming Transition and the Marginally Jammed Solid, Annu. Rev. Condens. Matter Phys. 1, 347 (2010).

[19] S. Torquato and F. H. Stillinger, Jammed Hard-Particle Packings: From Kepler to Bernal and Beyond, Rev. Mod. Phys. 82, 2633 (2010).

[20] T. Hayashi and R. W. Carthew, Surface Mechanics Mediate Pattern Formation in the Developing Retina, Nature (London) 431, 647 (2004).

[21] J. A. Åström and M. Karttunen, Cell Aggregation: Packing Soft Grains, Phys. Rev. E 73, 062301 (2006).
[22] J. R. Ellis, Macromolecular Crowding: Obvious but Underappreciated, Trends Biochem. Sci. 26, 597 (2001).

[23] J. Balbo, P. Mereghetti, D.-P. Herten, and R. C. Wade, The Shape of Protein Crowders Is a Major Determinant of Protein Diffusion, Biophys. J. 104, 1576 (2013).

[24] G. Wascher, H. Haussner, and H. Schumann, An Improved Typology of Cutting and Packing Problems, Eur. J. Oper. Res. 183, 1109 (2007).

[25] J. Bennell, G. Scheithauer, Y. Stoyan, and T. Romanova, Tools of Mathematical Modeling of Arbitrary Object Packing Problems, Ann. Oper. Res. 179, 343 (2010).

[26] J. Kepler, Strena seu de nive sexangula (1611); http://www .thelatinlibrary.com/kepler/strena.html.

[27] T. C. Hales, A Proof of the Kepler Conjecture, Ann. Math. 162, 1065 (2005).

[28] T. C. Hales and S. P. Ferguson, The Kepler Conjecture: The Hales-Ferguson Proof, edited by J.C. Lagarias (Springer-Verlag, New York, 2011).

[29] M. H. Huang and P.-H. Lin, Shape-Controlled Synthesis of Polyhedral Nanocrystals and Their Facet-Dependent Properties, Adv. Funct. Mater. 22, 14 (2012).

[30] C.-W. Liao, Y.-S. Lin, K. Chanda, Y.-F. Song, and M. H. Huang, Formation of Diverse Supercrystals from SelfAssembly of a Variety of Polyhedral Gold Nanocrystals, J. Am. Chem. Soc. 135, 2684 (2013).

[31] U. Betke and M. Henk, Densest Lattice Packings of 3-Polytopes, Comput. Geom. 16, 157 (2000).

[32] E. R. Chen, A Dense Packing of Regular Tetrahedra, Discrete Comput. Geom. 40, 214 (2008).

[33] S. Torquato and Y. Jiao, Dense Packings of the Platonic and Archimedean Solids, Nature (London) 460, 876 (2009).

[34] E. R. Chen, M. Engel, and S. C. Glotzer, Dense Crystalline Dimer Packings of Regular Tetrahedra, Discrete Comput. Geom. 44, 253 (2010).

[35] S. Gravel, V. Elser, and Y. Kallus, Upper Bound on the Packing Density of Regular Tetrahedra and Octahedra, Discrete Comput. Geom. 46, 799 (2011).

[36] J. de Graaf, R. van Roij, and M. Dijkstra, Dense Regular Packings of Irregular Nonconvex Particles, Phys. Rev. Lett. 107, 155501 (2011).

[37] P. F. Damasceno, M. Engel, and S. C. Glotzer, Crystalline Assemblies and Densest Packings of a Family of Truncated Tetrahedra and the Role of Directional Entropic Forces, ACS Nano 6, 609 (2012).

[38] A. Haji-Akbari, E. R. Chen, M. Engel, and S. C. Glotzer, Packing and Self-Assembly of Truncated Triangular Bipyramids, Phys. Rev. E 88, 012127 (2013).

[39] Y. Kallus, V. Elser, and S. Gravel, Dense Periodic Packings of Tetrahedra with Small Repeating Units, Discrete Comput. Geom. 44, 245 (2010).

[40] J. C. Lagarias, Mysteries in Packing Regular Tetrahedra, Notices Am. Math. Soc. 59, 1540 (2012).

[41] A. Donev, F. H. Stillinger, P. M. Chaikin, and S. Torquato, Unusually Dense Crystal Packings of Ellipsoids, Phys. Rev. Lett. 92, 255506 (2004).

[42] Y. Jiao, F. H. Stillinger, and S. Torquato, Optimal Packings of Superballs, Phys. Rev. E 79, 041309 (2009). 
[43] Y. Kallus and V. Elser, Dense-Packing Crystal Structures of Physical Tetrahedra, Phys. Rev. E 83, 036703 (2011).

[44] M. Marechal and M. Dijkstra, Phase Behavior and Structure of Colloidal Bowl-Shaped Particles: Simulations, Phys. Rev. E 82, 031405 (2010).

[45] T. Ras, R. Schilling, and M. Weigel, Regular Packings on Periodic Lattices, Phys. Rev. Lett. 107, 215503 (2011).

[46] A. P. Gantapara, J. de Graaf, R. van Roij, and M. Dijkstra, Phase Diagram and Structural Diversity of a Family of Truncated Cubes: Degenerate Close-Packed Structures and Vacancy-Rich States, Phys. Rev. Lett. 111, 015501 (2013).

[47] H. Minkowski, Geometrie der Zahlen (Teubner, Leipzig, 1896).

[48] H. Minkowski, Dichteste gitterförmige Lagerung kongruenter Körper, Nachr. K. Ges. Wiss. Göttingen, 311 (1904); H. Minkowskiin Gesammelte Abhandlungen Vol. II (Teubner, Berlin, 1911), pp. 3-42.

[49] G. Kuperberg and W. Kuperberg, Double-Lattice Packings of Convex Bodies in the Plane, Discrete Comput. Geom. 5, 389 (1990).

[50] P. G. Szabó, M. C. Markót, T. Csendes, E. Specht, L. G. Casado, and I. García, New Approaches to Circle Packing in a Square with Program Codes (Springer-Verlag, New York, 2007).

[51] E. R. Chen, Ph.D. Thesis, University of Michigan, 2010.

[52] See Supplemental Material at http://link.aps.org/ supplemental/10.1103/PhysRevX.4.011024 for more details on Bravais lattice vectors, volume and face areas of our polyhedra, our numeric algorithm, and intersection equations for all regions.
[53] B. Grünbaum and G. C. Shephard, Tilings and Patterns (W. H. Freeman, New York, 1987).

[54] We exclude the irreducible spheric triangle groups $\Delta_{p, 2,2}$ (axial dihedral, $D_{p h}$ ) because its intermediate polyhedra (polygonal prisms, polygonal bipyramids) are very anisotropic.

[55] A. Haji-Akbari, M. Engel, A. S. Keys, X. Zheng, R. G. Petschek, P. Palffy-Muhoray, and S. C. Glotzer, Disordered, Quasicrystalline and Crystalline Phases of Densely Packed Tetrahedra, Nature (London) 462, 773 (2009).

[56] P. F. Damasceno, M. Engel, and S. C. Glotzer, Predictive Self-Assembly of Polyhedra into Complex Structures, Science 337, 453 (2012).

[57] E. G. Gilbert, D. W. Johnson, and S. S. Keerthi, A Fast Procedure for Computing the Distance between Complex Objects in Three-Dimensional Space, IEEE Journal of robotics and automation 4, 193 (1988).

[58] Y. Kallus, V. Elser, and S. Gravel, Method for Dense Packing Discovery, Phys. Rev. E 82, 056707 (2010).

[59] E. Marcotte and S. Torquato, Efficient Linear Programming Algorithm to Generate the Densest Lattice Sphere Packings, Phys. Rev. E 87, 063303 (2013).

[60] S. C. Glotzer and M. J. Solomon, Anisotropy of Building Blocks and Their Assembly into Complex Structures, Nat. Mater. 6, 557 (2007).

[61] G. van Anders, N. K. Ahmed, D. Klotsa, M. Engel, and S. C. Glotzer, Unified Theoretical Framework for Shape Entropy in Colloids, arXiv:1304.7545.

[62] G. van Anders, N. K. Ahmed, R. Smith, M. Engel, and S. C. Glotzer, Entropically Patchy Particles, arXiv:1309.1187. 